QUARTERLY OF APPLIED MATHEMATICS

VOLUME LXVI, NUMBER 4

DECEMBER 2008, PAGES 633-658

S $0033-569 X(08) 01107-X$

Article electronically published on October 7, 2008

\title{
ON CONSTRUCTIVE COMPLEX ANALYSIS IN FINANCE: EXPLICIT FORMULAS FOR ASIAN OPTIONS
}

\author{
BY \\ MICHAEL SCHRÖDER \\ Keplerstraße 30, D-69469 Weinheim (Bergstraße), Germany
}

\begin{abstract}
This paper develops a two-stage approach to the explicit valuation of Asian options which stresses the rôle of complex analytic techniques in general, and special functions in particular. First, integral representations for their Black-Scholes values in terms of Hermite functions are obtained; this is based on a Laplace transform arising in the work of Yor, and proceeds by analytical inversion. Explicit formulas for these integrals are then derived as a second step; this is done by combining series and asymptotic expansions which have as terms at worst special functions such as the error integral. Numerical examples then illustrate how the results enable a benchmark valuation of these options.
\end{abstract}

1. Introduction. The paper explores the rôle of constructive complex analysis and special functions in finance. This comes from questions that originate from the use of averages of prices or rates. Such averaging is widespread, but it is most conspicuous in Asian options, the class of path-dependent options written on the arithmetic average of security prices. Providing protection, in particular, against strong price fluctuations in volatile or narrow markets, these options are widely traded. Mathematically, however, averaging poses a number of problems. First and foremost among these is the following. Having modelled the prices of the underlying securities as a stochastic process, one is immediately confronted with a new process, namely that obtained from averaging the given security price process. It comes as no surprise that the value of Asian options depends on the latter process, and that averaging alters the distributional properties of the starting security price process. This is the case, in particular, in the simplified but basic Black-Scholes framework of this paper, where security prices are assumed to follow geometric Brownian motion. The progress of valuing Asian options over the last twenty years is delineated and surveyed in [21] and [8, 10].

Received March 1, 2007.

2000 Mathematics Subject Classification. Primary 33Cxx, 91B28; Secondary 33F05, 41A58, 41A60, $44 \mathrm{~A} 10$.

Key words and phrases. Complex analytic methods and special functions in finance, Asian options, asymptotic expansions and series for Asian option valuation, parabolic cylinder functions, hypergeometric and confluent hypergeometric functions. 
Our paper develops a perspective on such explicitly given valuation problems for contingent claims, which is as time-honoured, and universally applicable, as it is new. This is done by stressing the use of complex analytic methods as follows: the contingent claim values to be computed furnish new classes of special functions and these should be studied by employing the concepts and methods of special functions theory.

For Asian options we turn this program into a two-stage approach as follows. In Section 4 we look at the value functionals of Asian options and, as a first step, represent these by integrals. Then in Section 6 , as a second step, we develop series and asymptotic expansions for the integrals of step one. This rationale will in general also provide new angles on special functions; in the present paper the resulting new angles are on, in particular, parabolic cylinder functions and hypergeometric functions.

The integral representation of Section 4 combines several lines of thought. The stochastic side of this problem has been extensively studied in particular by Dufresne, Yor and coworkers; by way of an enveloping construction, our integral is ultimately based on a Laplace transform that arises from their work, as summarized in the two books [34, 35]. As our analytic inversion shows, this enveloping construction results in a dimension reduction of the problem. This new feature is expressed by the fact that our representations now are single integrals of products of two functions. These two functions in the products are found to be parabolic cylinder functions. This then provides obvious starting points for the adaption of special functions methodology to render our integrals computable.

Applications of such methods have been consistently hindered by the stucture of the representations of the Asian option value functional derived hitherto. In Section 6 we give series and asymptotic expansions for this functional. While originally double or triple series, with special functions as terms, were to be expected here, we obtain single series. Moreover, there are explicit estimates for the respective approximation errors, and both our expansions and our estimates do not require functions more difficult than the error integral.

Furnishing a new angle on the more practical side of the problems as well, our results permit in particular reduction of the valuation of all Asian options to computing one single function. As we note in Section 7.1, this normalized value function, however, has to be computed to rather high accuracies in order to be useful. In Section 7.2 we therefore demonstrate how our results now permit computation of such benchmarks for Black-Scholes values of Asian options by way of computing benchmarks for these normalized values.

The valuation of Asian options has in fact been an active area of research and, complementing [26, Section 1], it seems apt to stress here the following: the multiplicity and the variety of approaches to Asian option valuation may just reflect the multiplicity and variety of its interconnections with mathematics.

Our paper gives concrete expression to such a new perspective. As explained in Section 6.8, our simplest formulas for computation, those of Section 6.3, draw on the most difficult special functions: the hypergeometric functions of Gauss and Kummer. Highly interesting from a conceptual point of view, these functions in fact determine the structure of Asian option values, as we established in [25], building on the work of Yor and 
Dufresne. It is such connections of a structural nature to which our findings ultimately give expression, and our results in fact go beyond option valuation itself.

2. Preliminaries. This section establishes the notation for two classes of special functions basic for the paper. This uses $\operatorname{Erfc}(z)=(2 / \sqrt{\pi}) \int[z, \infty) \exp \left(-s^{2}\right) d s$, the complementary error function.

2.1. Hermite functions. Referring to [14, Sections 10.2ff], the Hermite function $\mathrm{H}_{-\mu}$ of degree any complex $-\mu$ is defined by $H_{-\mu}(z)=2^{-\mu} \Psi\left(\frac{\mu}{2}, \frac{1}{2} ; z^{2}\right)$ on $\mathbf{C}$, the complex plane, as a specialization of the confluent hypergeometric function of the second kind $\Psi$. Hermite functions are thus entire functions of $\mu$ and $z$, and for $\operatorname{Re}(\mu)>0$ have the integral representation:

$$
H_{-\mu}(z)=(1 / \Gamma(\mu)) \int_{0}^{\infty} \exp \left(-u^{2}-2 z u\right) u^{\mu-1} d u, \quad z \in \mathbf{C} .
$$

By way of $\operatorname{Erfc}(z)=(2 / \sqrt{\pi}) \exp \left(-z^{2}\right) H_{-1}(z)$ they thus generalize the complementary error function. Hermite functions of degree any integer $n \geq 0$, on the other hand, coincide with the $n$th Hermite polynomial. Hermite functions are related to the parabolic cylinder functions $D_{\mu}$ of [11, Chapter VIII] by

$$
H_{-\mu}(z)=\exp \left(\frac{1}{2} z^{2}\right) 2^{-\mu / 2} D_{-\mu}(z \sqrt{2}),
$$

and to the Weber parabolic cylinder functions $U$ of [1, Chapter 19] by

$$
H_{-\mu}(z)=\exp \left(z^{2}\right) 2^{+\mu / 2} U\left(-\left(\mu+\frac{1}{2}\right), z \sqrt{2}\right) .
$$

2.2. The functions $G_{\xi}$. These functions are products of the Section 2.1 Hermite functions and weighted complementary error functions $\mathrm{WE}_{\omega}$. The latter functions depend on parameter triples $\omega=\left(\omega_{1}, \omega_{2}, \omega_{3}\right)$, where $\omega_{1}>0$ is any positive real and the other two $\omega_{m}$ are any complex numbers. For any complex $z$ they are then defined by:

$$
\mathrm{WE}_{\omega}(z)=\exp \left(\omega_{2} z\right) \operatorname{Erfc}\left(\omega_{1} z+\omega_{3} /\left(2 \omega_{1}\right)\right) .
$$

The functions $G$ depend on parameter sextuples $\xi=\left((\mu, \beta), \xi^{*}\right)$ with $\xi^{*}=\left(\xi_{1}, \xi_{2}, \xi_{3}, \xi_{4}\right)$, where $\beta>0$ and $\xi_{1}>0$ are any positive reals and the other four parameters are any complex numbers. For any complex $z$ they are then given by:

$$
G_{\xi}(z)=H_{-\mu}(-\beta \cosh (z)) g_{\xi^{*}}(z)
$$

with the definition

$$
g_{\xi^{*}}(z)=\exp \left(\left(\xi_{4} /\left(2 \xi_{1}\right)\right)^{2}\right)\left(\mathrm{WE}_{\left(\xi_{1}, \xi_{2}, \xi_{4}\right)}+\mathrm{WE}_{\left(\xi_{1}, \xi_{3},-\xi_{4}\right)}\right),
$$

or alternatively:

$$
G_{\xi}(z)=\exp \left(\left(\xi_{4} /\left(2 \xi_{1}\right)\right)^{2}\right) \sum_{\kappa \in K(\xi)} H_{-\mu}(-\beta \cosh (z)) \mathrm{WE}_{\kappa^{*}}(z),
$$

defining $K(\xi)$ to be the set consisting of the two quintuples $\kappa=\left(\mu, \beta, \kappa^{*}\right)$ with $\kappa^{*}=$ $\left(\xi_{1}, \xi_{2}, \xi_{4}\right)$ or $\kappa^{*}=\left(\xi_{1}, \xi_{3},-\xi_{4}\right)$. For any fixed complex $\mu$ and real $b>0$, in particular note the representation:

$$
G_{\xi(h, a)}(z)=H_{-\mu}(-\cosh (z) / \sqrt{2 b}) g_{\xi^{*}(h, a)}(z),
$$


where

$$
g_{\xi^{*}(h, a)}(z)=e^{a^{2} h / 2}\left(e^{a w} \operatorname{Erfc}\left(\frac{w}{\sqrt{2 h}}+\frac{a}{2} \sqrt{2 h}\right)+e^{-a w} \operatorname{Erfc}\left(\frac{w}{\sqrt{2 h}}-\frac{a}{2} \sqrt{2 h}\right)\right),
$$

on defining sextuples $\xi(h, a)=\left(\mu, 1 / \sqrt{2 b}, \xi^{*}(h, a)\right)$ with $\xi^{*}(h, a)=(1 / \sqrt{2 h}, a,-a, a)$.

3. Asian option valuation: statement of problem. This section assembles basic notions and facts about Asian options and their normalized valuation in the Black-Scholes model.

3.1. Black-Scholes setting. In this paper we work in the Black-Scholes framework using the risk-neutral approach to the valuation of contingent claims; see for example [13. Sections 4.1-4.3] or [27, Sections 14.2-14.3]. Recall there are two securities. First, a riskless security, a bond, whose price grows at the continuously compounding positive interest rate $r$. Then, a risky security whose price process $S$ is modelled using Brownian motion on a given probability space as follows. There is a uniquely determined probability measure $Q$ on this space, the risk neutral measure, such that for the associated standard $Q$-Brownian motion $B$ we have:

$$
S_{t}=S_{0} \exp \left(\left(\varpi-\frac{1}{2} \sigma^{2}\right)+\sigma B_{t}\right), \quad t \in[0, \infty) ;
$$

equivalently, $S$ solves the $\operatorname{SDE} d S_{t} / S_{t}=\varpi d t+\sigma d B_{t}$. Here the time-0 value $S_{0}$ of $S$ is a positive real, and the positive real constant $\sigma$ is the volatility of $S$. The specific form of the otherwise arbitrary constant $\varpi$ depends on the nature of the security modelled. For example if $S$ is a stock, it is the interest rate $r$ minus the pertinent dividend rate.

3.2. Asian options and their normalized valuation. In the setting of Section 3.1, this section formalizes the notion of Asian options stressing their normalized valuation. The European-style arithmetic-average Asian option written at time $t_{0}$, with maturity $T$ and fixed-strike price $K$, is then the contingent claim on the time interval $\left[t_{0}, T\right]$ with the following time- $T$ payoff: the maximum of 0 and the excess over $K$ of the average price $\left(1 /\left(T-t_{0}\right)\right) \int_{\left[t_{0}, T\right]} S_{u} d u$ over $\left[t_{0}, T\right]$. Defining $(x)_{+}=\max \{0, x\}$ for any real $x$, the value $C_{t}$ of the Asian option at any time $t$ in $\left[t_{0}, T\right]$ is thus given as the following expectation:

$$
C_{t}=e^{-r(T-t)} E\left[\left(\frac{1}{T-t_{0}} \int_{t_{0}}^{T} S_{u} d u-K\right)_{+} \mid \mathcal{F}_{t}\right],
$$

computed with respect to the Section 3.1 risk-neutral measure $Q$ and conditional on the time- $t$ information $\mathcal{F}_{t}$. Based on the modification in [6. Part I] of a technique in [12, normalize the valuation problem. Restart the Brownian motion in $S$ at time $t$, and then use its scaling property to construct the factorization:

$$
C_{t}=\frac{\exp (-r(T-t))}{T-t_{0}} \frac{S_{t}}{(\sigma / 2)^{2}} C^{(\nu)}(h, q) .
$$

The factorization is in terms of the integral of geometric Brownian motion, the process given by

$$
A_{w}^{(\nu)}=\int_{0}^{w} e^{2\left(B_{s}+\nu s\right)} d s, \quad w \in(0, \infty),
$$

and reduces the general valuation problem to computing the following expectation:

$$
C^{(\nu)}(h, q)=E\left[\left(A_{h}^{(\nu)}-q\right)_{+}\right],
$$


the normalized value of the Asian option. The point of this normalization is that the latter is now a dimensionless quantity given in terms of the dimensionless parameters:

$$
\nu=2 \varpi / \sigma^{2}-1 \quad \text { and } \quad h=(\sigma / 2)^{2}(T-t)
$$

Here $\nu$ is the normalized adjusted interest rate and $h$ is the normalized time to maturity, which is nonnegative. The exact form of the factorization is determined by the dimensionless parameter $q$ given, as an affine linear function of $h$ in particular, by:

$$
q=\frac{T-t_{0}}{(2 / \sigma)^{2} S_{t}}\left(K-\frac{1}{T-t_{0}} \int_{t_{0}}^{t} S_{u} d u\right)
$$

the normalized strike price. Indeed, if $q$ is nonpositive, Asian options lose their option feature, and, as has already been observed in [12, p. 361], their normalized price is given almost tautologically as follows.

FACT 3.1. If $q \leq 0$ we have $C^{(\nu)}(h, q)=E\left[A^{(\nu)}(h)\right]-q$ with the first moment of $A^{(\nu)}(h)$ given by $E\left[A^{(\nu)}(h)\right]=(\exp (2 h(\nu+1))-1) /(2(\nu+1))$ as an analytic function in $\nu$.

Valuing Asian options thus reduces to the case $q>0$, which is considered in the sequel.

4. Integral representations. In the setting of Sections 2 and 3, this section establishes integral representations for normalized values $C^{(\nu)}$ of Asian options as a first step in the study of these functions.

4.1. Statement and discussion of main results. The first main finding of this paper is an integral representation for the Section 3.2 normalized value $C^{(\nu)}(h, q)$ of Asian options in the generic case where $q>0$. It expresses this function as a single contour integral of products of two Section 2.1 Hermite functions $H_{-\mu}$ by way of the Section 2.2 functions $G_{\xi}$. Our result is as follows:

Theorem 4.1. If in the above setting $q=q(h)>0$ we have

$$
C^{(\nu)}(h, q)=\gamma_{\nu, q} \frac{\exp \left(-\nu^{2} h / 2\right)}{2 i} \int_{L}\left(G_{\xi(h, \nu+2)}-G_{\xi(h, \nu)}\right)(w) d w
$$

where

$$
\gamma_{\nu, q}=\frac{1}{2 \pi} \frac{\Gamma(\nu+4)}{\nu+1} \exp \left(-\frac{1}{2 q}\right)(2 q)^{\frac{\nu}{2}+1}
$$

and $L$ is any Figure 4.1 contour $L_{\theta, A}$ with $A \geq 0$ and $\xi(h, a)=\left(\nu+4,1 / \sqrt{2 q}, \xi^{*}(h, a)\right)$ for $\xi^{*}(h, a)=(1 / \sqrt{2 h}, a,-a, a)$.

To explain the remaining concepts, $L_{\theta, A}$ is, for any $\theta$ in $\left[\frac{\pi}{2}, \pi\right]$ and any real $A$, the contour of integration in the complex plane of Figure 4.1. 


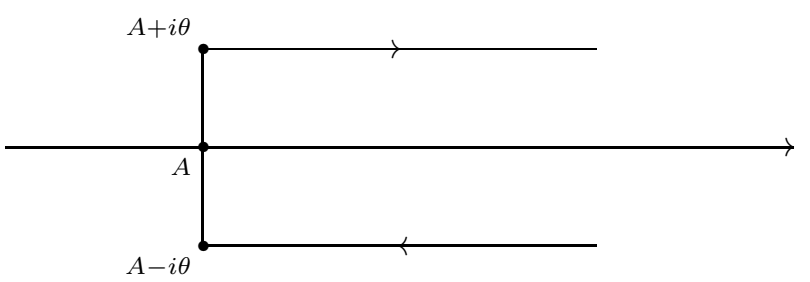

Figure 4.1. The contour $L_{\theta, A}$.

The functions $G_{\xi(h, a)}$ are the particular instances of the Section 3.2 functions $G_{\xi}$ given by

$$
G_{\xi(h, a)}(w)=H_{-\mu}(-\cosh (w) / \sqrt{2 q}) g_{\xi(h, a)}(w),
$$

for any complex $w$, where

$$
g_{\xi(h, a)}(w)=e^{a^{2} h / 2}\left(e^{a w} \operatorname{Erfc}\left(\frac{w}{\sqrt{2 h}}+\frac{a}{2} \sqrt{2 h}\right)+e^{-a w} \operatorname{Erfc}\left(\frac{w}{\sqrt{2 h}}-\frac{a}{2} \sqrt{2 h}\right)\right) .
$$

The proof of Theorem 4.1 is given in Section 4.7. Its starting point is a Laplace transform that ultimately goes back to Yor and coworkers, and proceeds by analytic inversion of the latter. Pertinent results are summarized in Sections 4.2 and 4.3 following the modifications in [6, 2003], a paper where an equivalent but different form of the Theorem 4.1 integral representation was announced and illustrated.

REMARK 4.2. The integral representation for $C^{(\nu)}$ of Theorem 4.1 is preceded by those representations obtained using any of the explicit forms of the law of $A^{(\nu)}$. Structurally, however, it is different from all of these. We look for this at those forms of the law which are obtained using the same approach as our result: the Hartman-Watson approach of 32. Here, three forms of the time- $h$ density $\lambda_{\nu, h}$ of $A^{(\nu)}$ have been obtained in 33, 9, and 23. They have been shown to be equivalent to each other, and express $\lambda_{\nu, h}$ as a single contour integral against Hermite function factors as follows:

$$
\lambda_{\nu, h}(w)=\Gamma(\nu+1) \frac{\alpha_{\nu, h}(w)}{2 \pi i} \int_{L} e^{-\frac{z^{2}}{2 h}} \sinh (z) H_{-(\nu+1)}\left(-\frac{\cosh (z)}{\sqrt{2 w}}\right) d z
$$

where

$$
\alpha_{\nu, h}(w)=\frac{2^{\nu / 2}}{\sqrt{\pi h}} \exp \left(-\frac{1}{2}\left(\nu^{2} h+\frac{1}{w}\right)\right) w^{(1 / 2)(\nu-1)-1} .
$$

Here $w$ is any real with $w>0$, and $L=L_{\theta, A}$ is any Figure 4.1 contour with $A \geq 0$. By way of $C^{(\nu)}(h, q)=\int_{0}^{\infty}(w-q)^{+} \lambda_{\nu, h}(w) d w$ we thus get normalized values as double integrals against Hermite functions. These differences give expression to the different approaches used: the above representation originates from a direct attack on the law, whereas the one of Theorem 4.1 is ultimately based on an indirect enveloping construction.

4.2. First reduction by an enveloping construction. At the heart of Theorem 4.1 is a simple observation about reducing dependencies on the time $h$ in the original Section 3.2 question. Indeed, the parameter $q$ there is by its definition a function of $h$, which is affine linear of the form $q(h)=k h+q^{*}$ with $k=K / S_{t}$. Discarding this dependency, define for any real $a>0$ functions $f_{G Y, a}$ on $[0, \infty)$ by:

$$
f_{G Y, a}(x)=E\left[\left(A_{x}^{(\nu)}-a\right)_{+}\right], \quad x \geq 0 .
$$


This yields a family of functions which, taken individually, cannot be used to value the original Asian option but as a whole allow recovery of $C^{(\nu)}$ as follows.

Lemma 4.3. If $q(h)>0$ we have $C^{(\nu)}(h, q)=\left.f_{G Y, a}\right|_{a=q(h)}(h)$.

This reduction leads to a study of these functions $f_{G Y, a}$ alone. As already explained in [6. Part I], these functions lack an interpretation as values of a traded option in general and hence are purely mathematical objects.

4.3. Laplace transforms. As a second step in establishing Theorem 4.1 this section recalls the Laplace transform of the Section 4.2 functions $f_{G Y, a}$. Here the Laplace transform is considered as a linear operator $\mathcal{L}$ on the continuous functions on $(0, \infty)$ of at most exponential growth. Any such function $f$ is thus mapped to the complex-valued function $\mathcal{L}(f)$ given by:

$$
\mathcal{L}(f)(z)=\int_{0}^{\infty} \exp (-z u) f(u) d u,
$$

for any complex $z$ in a half-plane contained sufficiently deep within the right-hand complex half-plane. The maps $\mathcal{L}(f)$ then are analytic on such half-planes, and $\mathcal{L}$ is injective with inverse $\mathcal{L}^{-1}$; see [2, Chapter 7] or [7.

The Laplace transform of any $f_{G Y, a}$ is then in terms of the modified Bessel functions $I_{\mu}$ of complex order $\mu$ (more fully discussed in [14, Chapter 5]) as follows.

Lemma 4.4. We have $f_{G Y, a}=\mathcal{L}^{-1}\left(F_{G Y, a}\right)$ for any real $a>0$, with $F_{G Y, a}$ defined by

$$
F_{G Y, a}(z)=\frac{D_{\nu, a}(z)}{z(z-2(\nu+1))}, \quad \operatorname{Re}(z)>\max \{0,2(\nu+1)\} .
$$

Here, on choosing the principal branch of the logarithm,

$$
D_{\nu, a}(z)=\frac{\exp (-1 /(2 a))}{a} \int_{0}^{\infty} e^{-x^{2} /(2 a)} x^{\nu+3} I_{\sqrt{2 z+\nu^{2}}}\left(\frac{x}{a}\right) d x .
$$

This is the adaption of [6. Parts II-VI], with three different proofs, of [12, Equation (3.9), p. 363]; for more on the latter paper's perspective, see also [35. A PDE proof of an equivalent result can be obtained along the lines of [16, p. 103f].

4.4. A gamma function interpretation of Bessel functions. With hindsight, it is the gamma function which is at the base of the analytical inversion of the Section 4.3 Laplace transform, and thus at the base of valuing the Asian option by way of Section 4.2. The connection comes via a classical integral representation, namely Hankel's formula:

$$
1 / \Gamma(s)=(1 /(2 \pi i)) \int_{C} \exp (\xi) \xi^{-s} d \xi
$$

valid for any complex $s$ and with $C$ any Hankel contour (see [31, p. 245]; typical such contours $C$ are the exponentials of the Figure 4.1 contours: $C=\exp \left(L_{\theta, A}\right)$ with $\theta$ in $\left(\frac{\pi}{2}, \pi\right]$ and $A$ any real. The Hankel formulas enter into the current problem by way of the series for the modified Bessel functions in Proposition 4.3. For any complex $\mu$ these are given in [14, Section 5.7] as:

$$
I_{\mu}(z)=\left(\frac{z}{2}\right)^{\mu} \sum_{m=0}^{\infty} \frac{1}{\Gamma(\mu+m+1)} \frac{1}{m !}\left(\frac{z}{2}\right)^{2 m}, \quad z \in \mathbf{C} \backslash \mathbf{R}_{<0} .
$$

The idea is to substitute for any reciprocal gamma factor of this series the corresponding Hankel formula and interchange the order of summation and contour integration; see 
[31, 17.231, p. 362], for instance. The result is the following classical contour integral representations on the right-hand half-plane.

Lemma 4.5. If $\operatorname{Re}(z)>0$ we have: $I_{\mu}(z)=(1 /(2 \pi i)) \int_{L} \exp (-\mu \xi+z \cosh (\xi)) d \xi$ for any contour $L=L_{\theta, A}$ with $\theta$ in $\left(\frac{\pi}{2}, \pi\right)$ and $A$ any real.

4.5. Dissecting Hermite functions. Using the Lemma 4.5 integral representation in Lemma 4.4 first yields, for any function $F_{G Y, a}$, their representation as a double integral:

$$
F_{G Y, a}(z)=4 \frac{e^{-1 /(2 a)}}{a} \int_{0}^{\infty} \int_{L_{\theta, A}} e^{-\frac{x^{2}}{2 a}} x^{\nu+3} e^{\frac{x}{a} \cosh (w)} \frac{\exp (-w \sqrt{\eta})}{\left(\eta-\nu^{2}\right)\left(\eta-(\nu+2)^{2}\right)} d w d x
$$

where $\eta=2 z+\nu^{2}$. Looking at the factor of the integrand depending on the variable $\eta$, the idea is to effect the Laplace inversion of this function by Laplace inversion under the double integral sign. Looking at the $\eta$-free factor of the integrand, from Section 2.1 it is essentially the integrand of the degree $-\mu$ Hermite function if $\mu=\nu+4>0$, and will yield this function after a change of variable if the order of the two integrals can be interchanged too. Writing $\mathcal{L}^{-1}$ as given by the contour integral of the Riemann inversion formula (see [2, Chapter 7, §5]) both questions reduce to the integrability of the threevariable integrand which thus results. Sufficient for this integrability is $\operatorname{Re}(w \sqrt{z})>0$, a condition which is seen to hold on choosing the contour parameter $A$ large enough. For any real $a>0$ and for $b$ equal to $\nu$ or $\nu+2$ define functions on $(0, \infty)$ by mapping any $h>0$ to

$$
\Sigma_{b}^{a}(h)=\frac{1}{2 \pi i} \int_{L_{\theta, A}} H_{-(\nu+4)}\left(-\frac{\cosh (w)}{\sqrt{2 a}}\right) \mathcal{L}^{-1}\left(\frac{\exp (-w \sqrt{z})}{z-b^{2}}\right)\left(\frac{h}{2}\right) d w,
$$

for any Lemma 4.5 contour $L_{\theta, A}$ with $A \gg 0$. Setting $c_{1}(a)=2 \pi \exp \left(-\frac{1}{2} \nu^{2} h\right) \gamma_{\nu, a}$ the effect of the gamma function on the Lemma 4.4 problem thus sums up as follows.

Lemma 4.6. If $\nu \geq 0$ we have $\mathcal{L}^{-1}\left(F_{G Y, a}\right)(h)=c_{1}(a)\left(\Sigma_{\nu+2}^{a}(h)-\Sigma_{\nu}^{a}(h)\right)$, where the functions $\Sigma_{b}^{a}$ are independent of the contour chosen and have integrable integrands.

With this result achieved, the proof of the Theorem 4.1 integral representation proceeds in two steps. As a first step, this result is established for $\nu \geq 0$ in the subsequent Section 4.6. As a second step, the validity of this finding is extended in Section 4.7 to any complex $\nu$.

4.6. Proof of Theorem 4.1 if $\nu$ is nonnegative. With $\nu \geq 0$ and $q>0$ successive application of Lemma 4.3, Lemma 4.4 and Lemma 4.6 results in:

$$
C^{(\nu)}(h, q)=\left.\mathcal{L}^{-1}\left(F_{G Y, a}\right)\right|_{a=q}(h)=c_{1}(q)\left(\Sigma_{\nu+2}^{q}(h)-\Sigma_{\nu}^{q}(h)\right),
$$

expressing the value function in terms of the Section 4.5 avatars $\Sigma_{b}^{q}$ of the gamma function. Further enlarging the parameters $A$ if necessary, assume there that the points of the contours $L_{\theta, A}$ as well as their squares are contained in the right-hand half-plane. A standard result from the theory of the heat equation then identifies the Laplace transform in $\Sigma_{b}^{q}$ in terms of the Section 2.2 functions $g_{\xi}$ as follows:

$$
2 \mathcal{L}^{-1}\left(\exp (-w \sqrt{z}) /\left(z-b^{2}\right)\right)(t)=g_{\xi(2 t, b)}(w) .
$$


For the $\Sigma_{b}^{q}$ we thus arrive at the contour integral representation:

$$
2 \Sigma_{b}^{q}(h)=(1 /(2 \pi)) \int_{L_{\theta, A}} g_{\xi(h, b)}(w) H_{-(\nu+4)}(-\cosh (w) / \sqrt{2 q}) d w,
$$

if $A \gg 0$. Using the analyticity of the integrand this last condition can be weakened to $A \geq 0$, and, similarly, $\theta$ can now be chosen from the closed interval $\left[\frac{\pi}{2}, \pi\right]$. Since the integrand is just the Section 2.2 function $G_{\xi(h, b)}$, as desired, the proof of Theorem 4.1 in the case $\nu \geq 0$ is complete.

4.7. Proof of Theorem 4.1 in the general case. As the second step of the proof of Theorem 4.1 we extend its validity from $\nu \geq 0$, as established in Section 4.6, to any complex $\nu$. For this, recall the entireness of Hermite functions in their degree. With the integral of Theorem 4.1 thus checked to be a meromorphic function in the variable $\nu$, the second step reduces to showing $C^{(\nu)}(h, q)$ to be an entire function in $\nu$. Indeed, since from Section 4.6 these two functions agree on nonnegative real numbers $\nu$, using the identity theorem they then agree on the complex plane as meromorphic functions. With one of them entire, the other one is then entire, too.

An extension of the value function to complex $\nu$, which is entire as well, has been provided in [6, $\S 17$ Lemma]. This is done by showing the absolute convergence of the power series expansion:

$$
\exp \left(\frac{1}{2} \nu^{2} h\right) C^{(\nu)}(h, q)=\sum_{n=0}^{\infty} \frac{\nu^{n}}{n !} E\left[f\left(A_{h}^{(0)}\right) B_{h}^{n}\right]
$$

for all complex $\nu$, where $f(x)=(x-q)_{+}$for any real $x$. The idea is as follows. The left-hand side is, for real $\nu$, equal to $E\left[f\left(A^{(0)}(h)\right) \exp \left(\nu B_{h}\right)\right]$ by a standard change of measure result, and our result thus asserts the following: on developing the exponential factor into its series, term-by-term integration of the resulting series makes sense, and is justified for complex $\nu$ as well. For this we apply the Cauchy-Schwarz inequality to each of its summands separately, and thus construct a majorizing series in terms of the moments of the Brownian motion $B_{h}$; this converges using the ratio test. The proof of Theorem 4.1 is complete.

5. Reductions. This section collects two preliminary properties of the contour integrals:

$$
(1 /(2 i)) \int_{L} G_{\xi}(\eta) d \eta
$$

as in Theorem 4.1. Here $L$ is any Figure 4.1 contour $L_{\theta, A}$ with $\theta$ in $\left[\frac{\pi}{2}, \pi\right]$ and any real $A \geq 0$. For the integrands more generally let $\xi=\left(\mu, \beta, \xi^{*}\right)$ with $\xi^{*}=(\alpha, a, b, c)$ being any complex parameter sextuple such that $\alpha$ and $\beta$ are positive reals.

5.1. First reduction. This section gives a real analysis transcription of the above contour integrals. More precisely, it expresses them as real integrals of the real part $\operatorname{Re}\left(G_{\xi}\right)$ and the imaginary part $\operatorname{Im}\left(G_{\xi}\right)$ of the function $G_{\xi}$ as follows.

Proposition 5.1. In the above setting we have:

$$
(1 /(2 i)) \int_{L_{\theta, A}} G_{\xi}(\eta) d \eta=\int_{0}^{\theta} \operatorname{Re}\left(G_{\xi}\right)(A+i \phi) d \phi+\int_{A}^{\infty} \operatorname{Im}\left(G_{\xi}\right)(y+i \theta) d y .
$$


This decomposition has two sources. First, the compatibility of $G=G_{\xi}$ with complex conjugation in the sense that $G(\bar{z})=\overline{G(z)}$. Second, the symmetry of the contour $L_{\theta, A}$ with respect to the real axis. An explicit computation then completes the proof.

5.2. Second reduction. With hindsight, it is the functions $G_{\xi}$ with not too small a negative degree Hermite function factors for which this paper's formulas work best, a fact relevant in particular for the option valuation problems in terms of the functions $G_{\xi(h, a)}$ established in Section 4. This section therefore gives recurrences which permit achieving this situation, at the cost, however, of not preserving this last class of functions.

To raise the indices $-\mu$ use:

$$
G_{\xi}=\frac{\beta / 2}{\mu-1} G_{\xi[\mu-1, a+1, b+1]}+\frac{\beta / 2}{\mu-1} G_{\xi[\mu-1, a-1, b-1]}+\frac{1 / 2}{\mu-1} G_{\xi[\mu-2]},
$$

and to lower the indices $-\mu$ use:

$$
G_{\xi}=-\beta G_{\xi[\mu+1, a+1, b+1]}-\beta G_{\xi[\mu+1, a-1, b-1]}+2(\mu+1) G_{\xi[\mu+2]} .
$$

To simplify notation we here denote changes in the parameters of $\xi$ by giving them in brackets after $\xi$; for example, $\xi[\mu+2]=\left(\mu+2, \beta, \xi^{*}\right)$. For proofs of these recurrences use the recurrences of [14, Equation (10.4.6)] for Hermite functions. Explicit formulas for $n$-fold iterations are in 22 , Chapter IV, $\S 5]$.

6. Series and asymptotic expansions. Working in the setting of Sections 4 and 5 , this section demonstrates how to use series and asymptotic expansions to develop for integrals such as those of Theorem 4.1 against the functions $G_{\xi}$ of Section 2.2 a framework of implementable formulas for their numerical computation. First we derive such formulas in Sections 6.3 to 6.4 when the Hermite functions in $G_{\xi}$ have negative degrees $-\mu$, and then appeal to the recurrences of Section 5.2 to reduce the general case to this situation; recall that the instability of the Theorem 4.1 functions $G_{\xi(h, a)}$ under these recurrences makes the more general functions $G_{\xi}$ necessary.

6.1. Formulaire. Complementing Section 2, this section collects further concepts and functions to be used in the sequel. Let $(\lambda)_{k}$ denote the $k$ th Pochhammer symbol of any complex $\lambda$, defined by $(\lambda)_{0}=1$ and $(\lambda)_{k+1}=(\lambda+k)(\lambda)_{k}$, for any integer $k \geq 0$.

6.1.1. Iterated weighted error functions IE. These functions are defined by integration of the functions

$$
f_{\alpha, \delta, z}(x) \stackrel{\text { def }}{=} e^{-z x} \operatorname{Erfc}(\alpha x+\delta)=\mathrm{WE}_{(\alpha,-z, 2 \alpha \delta)},
$$

for any real $\alpha>0$ and complex $\delta, z$ over any subinterval $[A, B)$ of the nonnegative reals as follows: $\operatorname{IE}_{J}(z)=\int_{[A, B)} f_{\alpha, \delta, z}(x) d x$ setting $J=(\alpha, \delta, A, B)$.

On partial integration,

$$
\begin{aligned}
\alpha \operatorname{IE}_{J}(0)= & (\alpha B+\delta) \operatorname{Erfc}(\alpha B+\delta)-(\alpha A+\delta) \operatorname{Erfc}(\alpha A+\delta) \\
& +(1 / \sqrt{\pi})\left(e^{-(\alpha A+\delta)^{2}}-e^{-(\alpha B+\delta)^{2}}\right),
\end{aligned}
$$

and for any complex $z$ different from zero,

$$
\begin{aligned}
z \operatorname{IE}_{J}(z)= & e^{-z A} \operatorname{Erfc}(\alpha A+\delta)-e^{-z B} \operatorname{Erfc}(\alpha B+\delta) \\
& +e^{\left(\delta+\frac{z}{2 \alpha}\right)^{2}-\delta^{2}}\left(\operatorname{Erfc}\left(\frac{z}{2 \alpha}+\delta+\alpha B\right)-\operatorname{Erfc}\left(\frac{z}{2 \alpha}+\delta+\alpha A\right)\right) .
\end{aligned}
$$


6.1.2. Iterated weighted error functions IEc. These depend on triples $J_{0}=(\alpha, \delta, B)$ with reals $\alpha>0, B \geq 0$ and complex $\delta$, and for any complex $z$ are defined by: $\operatorname{IEc}_{J_{0}}(z)=$ $\lim _{D \rightarrow \infty} \operatorname{IE}_{\left(J_{0}, D\right)}(z)$. Using the results of Section 6.1.1,

$$
\alpha \operatorname{IEc}_{J_{0}}(0)=(1 / \sqrt{\pi}) e^{-(\alpha B+\delta)^{2}}-(\alpha B+\delta) \operatorname{Erfc}(\alpha B+\delta)
$$

and for any complex $z$ different from zero,

$$
z \operatorname{IEc}_{J_{0}}(z)=e^{-z B} \operatorname{Erfc}(\alpha B+\delta)-e^{\left(\frac{z}{2 \alpha}+\delta\right)^{2}-\delta^{2}} \operatorname{Erfc}\left(\alpha B+\delta+\frac{z}{2 \alpha}\right) .
$$

6.1.3. Hermite function coefficients. An absolutely and compactly convergent series for the Section 2.1 Hermite functions $H_{-\mu}$ of degree any complex $-\mu$ is given by: $H_{-\mu}(z)=\sum_{k=0}^{\infty} a_{k} z^{k}$ with coefficients $a_{k}=a_{k}(\mu)$ equal to:

$$
a_{2 k}=\frac{2^{-\mu} \Gamma(1 / 2)}{\Gamma((1+\mu) / 2))} \frac{(\mu / 2)_{k}}{(1 / 2)_{k}} \frac{1}{k !} \quad \text { and } \quad a_{2 k+1}=\frac{2^{-\mu} \Gamma(-1 / 2)}{\Gamma(\mu / 2)} \frac{((1+\mu) / 2)_{k}}{(3 / 2)_{k}} \frac{1}{k !} .
$$

If $\mu$ is not a nonpositive integer, $a_{k}=(1 /(2 \Gamma(\mu))) \Gamma((k+\mu) / 2)\left((-2)^{k} / k\right.$ !); see [14, Section $10.4]$.

The asymptotics of $H_{-\mu}$ for large arguments in the right half-plane is described by the expansion: $H_{-\mu}(z)=\sum_{k=0}^{n-1} b_{k} /(2 z)^{2 k+\mu}+O\left(1 /|z|^{\operatorname{Re}(\mu)+2 n}\right)$, where

$$
b_{k}=b_{k}(\mu)=(-1)^{k}(\mu)_{2 k} / k !,
$$

which is valid for any complex $z$ with $|\arg (z)|<(3 / 4) \pi$.

6.1.4. The coefficients $\beta$. We let $\beta_{n, k}$ for any nonnegative integers $n$ and $k \leq n$ denote the weighted binomial coefficients given by:

$$
\beta_{n, k}=\frac{1}{2^{n}}\left(\begin{array}{l}
n \\
k
\end{array}\right) \quad \text { except that } \beta_{n, \frac{n}{2}}=\frac{1}{2^{n+1}}\left(\begin{array}{c}
n \\
n / 2
\end{array}\right)
$$

if $n$ is even and $k=n / 2$.

6.2. Basic framework. We address the computation of the Section 5 contour integrals of the Section 2.2 functions $G_{\xi}$ where $\xi=\left(\mu, \beta, \xi^{*}\right)$ with $\xi^{*}=\left(\alpha, \xi_{2}, \xi_{3}, \xi_{4}\right)$. This is by refining their Proposition 5.1 decomposition as follows:

$$
(1 /(2 i)) \int_{L} G_{\xi}(\eta) d \eta=\exp \left(\left(\xi_{4} /\left(2 \xi_{1}\right)\right)^{2}\right) \sum_{\kappa \in K(\xi)} R_{\kappa}(A)+I_{\kappa}(A, B)+I_{\kappa}(B, \infty) .
$$

To explain the additional concepts, here we first restrict ourselves to any contour $L=$ $L_{\pi / 2, A}$ with $A \geq 0$, and to the contour $L_{\pi / 2,0}$ in particular. To define the functions appearing above, let $\kappa=\left(\mu, \beta, \kappa^{*}\right)$ with $\kappa^{*}=(\alpha, a, c)$ be any of the two elements $\left(\mu, \beta, \alpha, \xi_{2}, \xi_{4}\right)$ and $\left(\mu, \beta, \alpha, \xi_{3},-\xi_{4}\right)$ of the Section 2.2 set $K(\xi)$. For any reals $A \leq B$ in $[0, \infty]$ put:

$$
\begin{aligned}
& I_{\kappa}(A, B)=\int_{A}^{B} \operatorname{Im}\left(H_{-\mu}(-i \beta \sinh (y)) \mathrm{WE}_{\kappa^{*}}(y+i \pi / 2)\right) d y, \\
& R_{\kappa}(A)=\int_{0}^{\pi / 2} \operatorname{Re}\left(H_{-\mu}(-\beta \cosh (A+i \phi)) \mathrm{WE}_{\kappa^{*}}(A+i \phi)\right) d \phi
\end{aligned}
$$

with WE denoting the Section 2.2 weighted complementary error functions.

Computation of the integrals $\int_{L} G_{\xi}(\eta) d \eta$ is thus reduced to computing three types of integrals $R_{\kappa}$ and $I_{\kappa}$ for any choice of $A \leq B$ in $[0, \infty]$. This we address below. 
6.3. Formulas for the real parts. Working in the setting of Section 6.2, this section concentrates on the total real part contributions $\bar{R}$. Specializing to any parameter quintuple $\kappa_{0}=(\mu, \beta, \alpha, a, c)$ with $a$ and $\mu$ real, these denote the four-term sums:

$$
\bar{R}_{\kappa_{0}}(A)=\sum_{\kappa \in L\left(\kappa_{0}\right)} R_{\kappa}(A)
$$

for any real $A \geq 0$. Here the summation runs over the set $L\left(\kappa_{0}\right)$ that consists of the four quintuples $(\mu, \beta, \alpha, \pm a, \pm c)$. For $A=0$ we simplify notation to $\bar{R}=\bar{R}(0)$.

The starting point is furnished by a representation in terms of Kummer's confluent hypergeometric functions $\Phi$; see for example [14, Sections 9.9-9.13] for more detail on these. Our precise result, proved in Section 6.8.2, is as follows.

Theorem 6.1. If in the above setting $\mu$ and $a+\mu$ are positive, then

$$
\bar{R}_{\kappa_{0}}=2 \pi \beta^{a} \frac{\Gamma((a+\mu) / 2)}{\Gamma(\mu) \Gamma(a+1)} \Phi\left(\frac{1}{2}(a+\mu), a+1 ; \beta^{2}\right)-4 K_{\lambda\left(\kappa_{0}\right)},
$$

where we associate with $\kappa_{0}$ the quadruple $\lambda\left(\kappa_{0}\right)=(\mu, \beta,(\alpha, a))$ and set

$$
K_{\lambda\left(\kappa_{0}\right)}=\int_{0}^{\infty} \operatorname{Im}\left(H_{-\mu}(-i \beta \sinh (y)) \exp \left(-a\left(y+i \frac{\pi}{2}\right)\right)\right) d y .
$$

If these error terms $K_{\lambda}$ are small, Theorem 6.1 yields an almost closed form for $\bar{R}$. We thus seek estimates for their size. We have found them as terms of the hypergeometric function generically; see Section 6.8.3. The present situation, however, requires only particular cases of our results; as we show in Section 6.8.4 the hypergeometic functions here become expressible in terms of elementary functions as follows.

TheOREM 6.2. If in the above setting $\mu>0$ and $a$ are such that $a+\mu>0$ and $\mu-a$ is any positive even integer, then we have

$$
\left|K_{\lambda\left(\kappa_{0}\right)}\right| \leq \frac{\Gamma(\mu / 2)}{\Gamma(\mu)} \frac{1-e^{-B a}}{a}+D_{a, \mu}(B),
$$

where

$$
D_{a, \mu}(B)=\frac{(\sqrt{2} / \beta)^{\mu}}{a+\mu} \sum_{n=0}^{M} \frac{(-M)_{n}}{(P)_{n}} \frac{\exp (-(\mu+a+2 n) B)}{\left(1-e^{-2 B}\right)^{\mu-1}},
$$

for any $B>0$, while $M=(\mu-a) / 2-1$ and $P=(\mu+a) / 2+1$.

To demonstrate the significance of these results in the option valuation situation of Theorem 4.1, define the total real parts contribution $R$ by the weighted difference:

$$
2 R=\exp (2 h(\nu+1)) \bar{R}_{\kappa(h, \nu+2)}-\bar{R}_{\kappa(h, \nu)},
$$

where $\kappa(h, c)=(\nu+4,1 / \sqrt{2 q}, 1 / \sqrt{2 h}, c, c)$. The contribution of the real parts to $C^{(\nu)}(h, q)$ is then obtained on multiplication of $R$ with the Theorem 4.1 function $\gamma_{\nu, q}$. The point is that, at least if $\nu$ is not too small, the brunt of this contribution becomes measurable in terms of elementary functions. Indeed, recalling the standard relation $\Phi(a, a, z)=\exp (z)$ the two theorems above can be checked to yield the following result.

COROllary 6.3. If $b>0$ and $\nu>-2$, then we have for $R$ the decomposition:

$$
R=R_{\mathrm{est}}+\Delta, \quad \text { where } \quad \gamma_{\nu, q} R_{\mathrm{est}}=\frac{1}{2(\nu+1)}\left(e^{2 h(\nu+1)}-(1+2 q(\nu+1))\right) .
$$


Here $\Delta=-2\left(\exp (2 h(\nu+1)) K_{\lambda(\nu+2)}-K_{\lambda(\nu)}\right)$ with $\lambda(\omega)=(\nu+4,1 / \sqrt{2 q}, 1 / \sqrt{2 h}, \omega)$ and measures the approximation error of this quasi-closed form $R_{\text {est }}$ to $R$.

6.4. Formulas for the imaginary parts. Working in the setting of Section 6.2, this section develops a framework of results for computing the imaginary part functions $I_{\kappa}(A, \infty)$ to any prescribed error of $2 \varepsilon>0$. This is based on the decomposition

$$
I_{\kappa}(A, \infty)=I_{\kappa}(A, B)+I_{\kappa}(B, \infty),
$$

valid for any $A \leq B$, and the principal idea is to proceed in two steps as follows. First, with dependence on $\kappa$, vary the break point $B \geq A$ so that $I_{\kappa}(B, \infty)$ can be computed up to an error of $\varepsilon$ by an asymptotic expansion. Then, as a second step, compute $I_{\kappa}(A, B)$ up to an error of $\varepsilon$ using a series. Both computations need explicit estimates for the approximation errors of the pertinent expansions, and have to be based on these estimates.

We restrict our discussion of the first step to Hermite functions of indices $-\mu$ with negative real parts; recall from Section 5.2 that the general case can be reduced to this setting using recurrence rules. Our asymptotic expansion is as follows.

TheOREM 6.4. If in the above setting $\mu_{0}=\operatorname{Re}(\mu)$ and $B$ are positive, then we have for any integer $n_{0} \geq 0$ the expansion

$$
I_{\kappa}(B, \infty)=\sum_{n=0}^{n_{0}-1} \frac{(-1)^{n} b_{n}}{\beta^{2 n+\mu}} \operatorname{Im}\left(e^{i \frac{\pi}{2}(\mu+a)} A_{\gamma, 2 n+\mu}(B)\right)+\rho_{n_{0}},
$$

where $\gamma=\gamma_{c}+i(\pi / 2) \alpha$ with $\gamma_{c}=c /(2 \alpha)$, and the error terms $\rho_{n_{0}}$ satisfy

$$
\left|\rho_{n_{0}}\right| \leq \exp \left((\alpha \pi)^{2} / 4\right) \frac{\Gamma\left(\mu_{0}\right)}{|\Gamma(\mu)|}\left|b_{n_{0}}\left(\mu_{0}\right)\right|(\sqrt{2} / \beta)^{2 n_{0}+\mu_{0}} A_{\gamma_{c}, 2 n_{0}+\mu_{0}}(B) .
$$

Here $b_{n}=b_{n}(\mu)$ and $b_{n}\left(\mu_{0}\right)$ are the coefficients of Section 6.1.3, and the terms $A_{\delta, \lambda}(B)$, for any complex $\delta$ and $\lambda$, are series in the Section 6.1.2 functions IEc as follows:

$$
A_{\delta, \lambda}(B)=\sum_{k=0}^{K}(-1)^{k}\left(\begin{array}{c}
-\lambda \\
k
\end{array}\right) \operatorname{IEc}_{(\alpha, \delta, B)}(\lambda-a+2 k)+R_{\delta, \lambda, K+1} .
$$

Here the remainder terms $R_{\delta, \lambda, K+1}$ satisfy:

$$
\left|R_{\delta, \lambda, K+1}\right| \leq\left|1-\frac{1}{\left(1-e^{-2 B}\right)^{\lambda}}\right|\left|\left(\begin{array}{c}
-(\lambda+1) \\
K
\end{array}\right)\right| e^{\operatorname{Im}^{2}(\delta)} \operatorname{IEc}_{(\alpha, \operatorname{Re}(\delta), B)}(\operatorname{Re}(\lambda-a)+2 K) .
$$

REMARK 6.5. A weakening of the Theorem 6.4 error estimate is:

$$
\left|\rho_{n_{0}}\right| \leq \exp \left((\alpha \pi)^{2} / 4\right) \frac{\Gamma\left(\mu_{0}\right)}{|\Gamma(\mu)|}\left|b_{n_{0}}\left(\mu_{0}\right)\right|(\sqrt{2} / \beta)^{2 n_{0}+\mu_{0}} \operatorname{IEc}_{\left(\alpha, \gamma_{c}, B\right)}\left(2 n_{0}+\mu_{0}-\operatorname{Re} a\right) .
$$

This again illustrates how the asymptotic expansions work better the bigger $\beta$ is.

Proofs of these results are in Section 6.7.2. Their basic idea, developed in Section 6.7.1 in greater generality and with all its technicalities, is: expand the Hermite function factors in $I_{\kappa}$ using the asymptotic expansion of Section 6.1.3 and integrate term-by-term.

This particular asymptotic expansion is known to work well for Hermite functions of degrees with not too large absolute values. Again such a situation can be achieved using the recurrences of Section 5.2. A large number of alternative asymptotic expansions has been studied, in part with complementary properties; see for instance those of 20 , 
Chapter 11, $\S 4,7,11],[18$, or [19] based on an asymptotic study of the solutions of second order ordinary differential equations, or those of [28] or 29] extending Olver's results. Applying such results and methods to $I_{\kappa}(B, \infty)$ seems to offer a wealth of questions which merit further study.

The idea for getting a series for the terms $I_{\kappa}(A, B)$ is to expand the Hermite function factors of their integrands into their series and integrate term-by-term. This is formalized in Section 6.6.1 and shown in Section 6.6.2 to result in the following series expansion with coefficients in terms of the Section 6.1.1 weighted error functions $\mathrm{IE}_{J}$.

ThEOREM 6.6. In the above setting, an absolutely convergent series for the $I_{\kappa}(A, B)$ is given, for any integer $n_{0} \geq 0$, by

$$
I_{\kappa}(A, B)=\sum_{n=0}^{n_{0}-1}(-\beta)^{n} a_{n} \sum_{k=0}^{\left[\frac{n}{2}\right]} \beta_{n, k}\left(w_{\gamma, a+n-2 k}^{*}+w_{\gamma, a-(n-2 k)}^{*}\right)+\rho_{n_{0}},
$$

where $\gamma=\gamma_{c}+i(\pi / 2) \alpha$ with $\gamma_{c}=c /(2 \alpha)$. If $\mu_{0}=\operatorname{Re}(\mu)>0$ the error terms $\rho_{n_{0}}$ satisfy:

$$
\left|\rho_{n_{0}}\right| \leq \exp \left((\alpha \pi)^{2} / 4\right)\left|a_{n_{0}}\left(\mu_{0}\right)\right| \beta^{n_{0}} \sum_{k=0}^{\left[n_{0} / 2\right]}(-1)^{k} \beta_{n_{0}, k} \Delta_{n_{0}, k} .
$$

Here $a_{n}=a_{n}(\mu)$ and $a_{n}\left(\mu_{0}\right)$ are the Hermite function coefficients of Section 6.1.3 and the $\beta_{n, k}$ are the weighted binomial coefficients of Section 6.1.4. In terms of the Section 6.1.1 functions $\mathrm{IE}_{J}$ we first put for any complex $\eta$,

$$
w_{\gamma, \eta}^{*}=\operatorname{Im}\left(e^{i \frac{\pi}{2} \eta} \operatorname{IE}_{J(\gamma)}(-\eta)\right)
$$

and

$$
\Delta_{n_{0}, k}=\operatorname{IE}_{J\left(\gamma_{c}\right)}\left(-\left(\operatorname{Re} a+n_{0}-2 k\right)\right)+(-1)^{n_{0}} \operatorname{IE}_{J\left(\gamma_{c}\right)}\left(n_{0}-2 k-\operatorname{Re} a\right),
$$

setting $J(\delta)=(\alpha, \delta, A, B)$ for any complex $\delta$.

REMARK 6.7. A simpler majorization of the Theorem 6.6 error terms is:

$$
\left|\rho_{n_{0}}\right| \leq \exp \left((\alpha \pi)^{2} / 4\right)\left|a_{n_{0}}\left(\mu_{0}\right)\right|(\beta / 2)^{n_{0}} \operatorname{IE}_{J\left(\gamma_{c}\right)}\left(-\left(\operatorname{Re} a+n_{0}\right)\right) .
$$

However, this estimate is in general coarser than that of the theorem.

Remark 6.8. It is tempting to let $B$ grow to $\infty$ in the Theorem 6.7 series and try to compute this limit term-by-term. However, this does not seem to be justified: the series thus obtained seem to diverge. Cutting off an improper integral first, as we did using asymptotic expansions, seems indeed to be vital for the validity of the approach.

6.5. Hermite functions made effective. We establish this section's results in three stages, each of a different nature. These correspond to the three classes of real integrals furnished by the Section 6.2 contour integrals; identifying these classes, the next three sections develop principal methods to represent their members by series or asymptotic expansions.

Making effective the Section 6.1.3 expansions for Hermite functions $H_{-\mu}$ of degree any complex $-\mu$ is a preliminary to our results. This section shows how to achieve this when $\mu_{0}=\operatorname{Re}(\mu)>0$. So consider the series and asymptotic expansions up to order any integer $n \geq 0$ as given by

$$
H_{-\mu}(z)=\sum_{k=0}^{n-1} a_{k} z^{k}+r_{n}(z) \quad \text { and } \quad H_{\mu}(z)=\sum_{k=0}^{n-1} \frac{b_{k}}{(2 z)^{2 k+\mu}}+R_{n}(z) \text {, }
$$


respectively with coefficients $a_{k}=a_{k}(\mu)=(1 /(2 \Gamma(\mu))) \Gamma((k+\mu) / 2)\left((-1)^{k} / k !\right)$ in the series and $b_{k}=b_{k}(\mu)=(-1)^{k}(\mu)_{2 k} / k$ ! in the asymptotic expansion. For the $n$ th-order error terms $r_{n}$ and $R_{n}$ we then have explicit estimates as follows.

Proposition 6.9. In the above setting, for any complex $z$ with $\operatorname{Re}(z) \geq 0$, we have

$$
\left|r_{n}(z)\right| \leq \frac{\Gamma\left(\mu_{0}\right)}{|\Gamma(\mu)|}\left|a_{n}\left(\mu_{0}\right)\right||z|^{n} .
$$

Proposition 6.10. In the above setting, for any complex $z$ with $\operatorname{Re}(z) \geq 0$, we have

$$
\left|R_{n}(z)\right| \leq \frac{\Gamma\left(\mu_{0}\right)}{|\Gamma(\mu)|}\left|b_{n}\left(\mu_{0}\right)\right|\left(\frac{\sqrt{2}}{2|z|}\right)^{2 n+\mu_{0}} .
$$

Omitting the somewhat lengthy details, the Proposition 6.9 estimate is found, rendering effective [14, Section 10.5]. The estimate of Proposition 6.10 can be checked rendering effective [14, Section 10.6]; this however is by now using there the representations $H_{-\mu}(z)=(1 / \Gamma(\mu)) \int_{\left[0, e^{i \theta} \infty\right]} \exp \left(-w^{2}-2 w z\right) w^{\mu-1} d w$ as contour integrals, with suitable choices of $|\theta|<\pi / 4$.

6.6. Proving the series for the imaginary parts. As a first step in proving the results, this section develops a general series expansion for Section 6.2 type integrals over finite horizontal parts of the Section 6.2 contours $L$, and shows how the Theorem 6.6 series for the incomplete imaginary parts follows from it.

6.6.1. A general series expansion. This section demonstrates a method for obtaining series expansions of integrals against special functions by proving a series expansion for the following integrals:

$$
I_{A, B}(f)=\int_{A}^{B} H_{-\mu}(-i \beta \sinh (y)) f(y) d y .
$$

Here $A, B \geq 0$ are any reals, $f$ is any continuous function on the subinterval $[A, B]$ of the nonnegative real line, $\mu$ is any complex variable with real part $\mu_{0}=\operatorname{Re}(\mu)$ and $\beta>0$ is any real. We proceed by reduction to the integrals given by

$$
\Delta_{A, B, a}^{n}(f)=I_{A, B, a}(f)+(-1)^{n} I_{A, B,-a}(f),
$$

where

$$
I_{A, B, a}(f)=\int_{A}^{B} e^{a y} f(y) d y,
$$

for any integer $n$ and any complex $a$. Our precise result is as follows.

ThEOREm 6.11. In the above setting, we have the absolutely convergent series, which for any integer $n_{0} \geq 0$ is given by:

$$
I_{A, B}(f)=\sum_{n=0}^{n_{0}-1} a_{n} \beta^{n} \sum_{k=0}^{[n / 2]} \exp \left(-i \frac{\pi}{2}(n+2 k)\right) \beta_{n, k} \Delta_{A, B, n-2 k}^{n}(f)+\rho_{n_{0}}(f) .
$$

Here the error terms $\rho_{n_{0}}(f)$ under the additional assumption $\mu_{0}>0$ satisfy:

$$
\left|\rho_{n_{0}}(f)\right| \leq \frac{\Gamma\left(\mu_{0}\right)}{|\Gamma(\mu)|}\left|a_{n_{0}}\left(\mu_{0}\right)\right| \beta^{n_{0}} \sum_{k=0}^{\left[n_{0} / 2\right]} \beta_{n, k}(-1)^{k} \Delta_{A, B, n_{0}-2 k}^{n_{0}}(|f|) .
$$

The proof of this result is in two steps. As a first step expand the Hermite function factor of the integrand of $I_{A, B}(f)$ into the series of Proposition 6.10, which is compactly 
convergent, and integrate term-by-term. Using a form of the Lebesgue Dominated Convergence theorem, the result is the absolutely convergent series:

$$
I_{A, B}(f)=\sum_{n=0}^{n_{0}-1} a_{n}(-i \beta)^{n} \int_{A}^{B} \sinh ^{n}(y) f(y) d y+\rho_{n_{0}}(f)
$$

whose remainder terms $\rho_{n_{0}}(f)=\int_{[A, B]} r_{n_{0}}(-i \beta \sinh (y)) f(y) d y$ go to 0 with $n_{0}$ tending to $\infty$. As a final step take care of the powers of the hyperbolic sine using:

$$
\sinh ^{n}(y)=\sum_{k=0}^{[n / 2]} \beta_{n, k}(-1)^{k}\left(e^{(n-2 k) y}+(-1)^{n} e^{-(n-2 k) y}\right) .
$$

To establish the estimate for the error term, first majorize the absolute value of $\rho_{n_{0}}(f)$ by taking the absolute value under the defining integral sign. Take the absolute value of the $r_{n_{0}}$ factor of the resulting integrand and then majorize using the estimate of Proposition 6.10. A reduction is thus checked to occur to computing the above integrals against $n$th powers of $\sinh$ for $n=n_{0}$ and with $f$ replaced by $|f|$. The proof of Theorem 6.12 is complete.

6.6.2. Proof of Theorem 6.6. Establishing the series of Theorem 6.6 for the Section 6.2 incomplete imaginary parts $I_{\kappa}(A, B)$ is done by reduction to the results of Section 6.6.1. Comparing definitions in Sections 2.2 and 6.1.1, first we have for the $\mathrm{WE}_{\kappa^{*}}$ factors of the integrands the identities:

$$
\mathrm{WE}_{\kappa^{*}}(y+i(\pi / 2))=e^{i a \frac{\pi}{2}} \exp (a y) \operatorname{Erfc}(\alpha y+\gamma)=e^{i a \frac{\pi}{2}} f_{\alpha, \gamma,-a}(y),
$$

recalling $\kappa^{*}=(\alpha, a, c)$ and $\gamma=\gamma_{c}+i(\pi / 2)$ with $\gamma_{c}=c /(2 \alpha)$. This permits expression of the incomplete imaginary parts in terms of the Section 6.6.1 integrals as follows:

$$
I_{\kappa}(A, B)=\operatorname{Im}\left(e^{i \frac{\pi}{2} a} I_{A, B}\left(f_{\alpha, \gamma,-a}\right)\right) .
$$

Applying Theorem 6.11, it is therefore sufficient to identify the integrals:

$$
I_{A, B, m}\left(f_{\alpha, \gamma,-a}\right)=\int_{A}^{B} \exp ((a+m) y) \operatorname{Erfc}(\alpha y+\gamma) d y
$$

as equal to the Section 6.1.1 function $\operatorname{IE}_{J}$ with $J=(\alpha, \gamma, A, B)$ at $z=-(a+m)$. This holds identically again. On checking how the signs in the Theorem 6.11 series are absorbed by the weighting roots of unity in $w_{J}^{*}$, the proof of Theorem 6.6 is complete.

6.7. Proving the asymptotic expansions. As a second step in proving the results, this section establishes the asymptotic expansion for the Theorem 6.4 complementary incomplete imaginary parts. This is seen as a consequence of a more general framework addressing expansions of Section 6.2 type integrals over unbounded horizontal parts of the Section 6.2 contours $L$ and that is developed first.

6.7.1. A general asymptotic expansion. This section demonstrates a method for obtaining two-step asymptotic expansions of integrals against special functions. For any complex variable $\mu$ with real part $\mu_{0}=\operatorname{Re}(\mu)>0$ and any real $\beta>0$, let $f$ be any continuous complex-valued function on any interval between $A+i \pi / 2$ and $B+i \pi / 2$ of the complex plane with $A, B$ in $[0, \infty]$ such that the following integrals exist:

$$
\begin{aligned}
& J(f)=J_{A, B}(f)=\int_{A+i \pi / 2}^{B+i \pi / 2} H_{-\mu}(-\beta \cosh (w)) f(w) d w, \\
& J_{c}(f)=J_{A, B, c}(f)=\int_{A}^{B} f\left(y+i \frac{\pi}{2}\right)(2 \sinh (y))^{-c} d y,
\end{aligned}
$$


for any complex number $c$ with $\operatorname{Re}(c) \geq 0$. As a first step, the computation of $J(f)$ is reduced to the computation of particular integrals $J_{c}(f)$ as follows.

Proposition 6.12. For $\mu$ any complex variable with real part $\mu_{0}=\operatorname{Re}(\mu)>0$, we have for any integer $N \geq 0$ the expansion:

$$
J(f)=e^{i \mu \frac{\pi}{2}} \sum_{n=0}^{N-1}(-1)^{n} \frac{b_{n}}{\beta^{2 n+\mu}} J_{2 n+\mu}(f)+\rho_{N}(f),
$$

where the coefficients $b_{n}=b_{n}(\mu)$ are as in Section 6.1 .3 and their error terms $\rho_{N}$ satisfy:

$$
\left|\rho_{N}(g)\right| \leq \frac{\Gamma\left(\mu_{0}\right)}{|\Gamma(\mu)|}\left|b_{N}\left(\mu_{0}\right)\right|(\sqrt{2} / \beta)^{2 N+\mu_{0}} J_{2 N+\mu_{0}}(g),
$$

for any function $g$ such that $|f(y+i(\pi / 2))| \leq g(y)$ on $[A, B]$.

Proof. This results from expanding the Hermite function factor of $J(f)$ into its asymptotic expansion of Section 6.5. More precisely, parametrizing in the defining integral $w=y+i \pi / 2$, so that $d w=d y$, we have $\cosh (y+i \pi / 2)=i \sinh (y)$. Thus apply the Section 6.5 asymptotic expansion with $z=-i \beta \sinh (y)$. Raising the factors $-i$ to $-(2 n+\mu)$-th powers yields the factors $\exp (i \pi(n+\mu / 2))$, and hence the expansion. The majorization of the error term $\rho_{N}$ can be checked to follow similarly using the explicit error estimate of Proposition 6.10. The proof of Proposition 6.12 is complete.

As a second step in computing $J(f)$, the idea is to establish conditions on the growth behaviour of $f$ under which there is a reduction in the computation of $J_{c}(f)$ to the integrals:

$$
J_{c, k}(f):=J_{A, B, c, k}(f)=\int_{A}^{B} \exp (-(c+2 k) y) f\left(y+i \frac{\pi}{2}\right) d y,
$$

for any integer $k \geq 0$. While we have $J_{c}(f)=J_{c, 0}(f)$ for $c=0$, for nonzero $c$, the integrals $J_{c}(f)$ are expressed by a series in the $J_{c, k}(f)$ as follows.

Proposition 6.13. Let $c \neq 0$ and $\beta$, with $\beta+c<0$, be any reals such that $J_{c, K}(|f|)$ divided by $K^{\beta}$ goes to 0 , as $K$ tends to $\infty$. If moreover $A>0$, then we have for $J_{c}(f)$ the absolutely convergent series given by

$$
J_{c}(f)=\sum_{k=0}^{K}(-1)^{k}\left(\begin{array}{c}
-c \\
k
\end{array}\right) J_{c, k}(f)+R_{c, K+1},
$$

for any integer $K \geq 0$, whose remainder terms $R_{c, K}$ satisfy

$$
\left|R_{c, K+1}\right| \leq\left|1-\frac{1}{\left(1-e^{-2 A}\right)^{c}}\right|\left|\left(\begin{array}{c}
-(c+1) \\
K
\end{array}\right)\right| J_{c, K}(|f|) .
$$

REMARK 6.14. We have $J_{c, K}(|f|) \leq J_{c, K}(g)$, for any function $g$ on the interval from $A+i \pi / 2$ to $B+i \pi / 2$ for which $|f| \leq g$ and $\eta \mapsto g(\eta) \exp (-(c+2 K) \eta)$ is integrable. Then the conclusion of Proposition 6.13 holds if there is a $\beta$ with $\beta+c<0$ such that $J_{c, K}(g)$ divided by $K^{\beta}$ goes to 0 as $K$ tends to $\infty$.

Proof of Proposition 6.13. In the denominator of the $J_{c}(f)$ integrand, note $2 \sinh (y)=$ $\exp (+y)(1-\exp (-2 y))$ and expand the resulting minus $c$ th power of the second factor into its binomial series. Any $K$ th-order error term of the series thus obtained is given by:

$$
R_{c, K}=\int_{A}^{B} \exp (-c y) f\left(y+i \frac{\pi}{2}\right) \rho_{K}(-\exp (-2 y)) d y
$$


where $\rho_{K}$ is the $K$ th-order error term of the binomial series:

$$
\rho_{K}(z)=K\left(\begin{array}{c}
-c \\
K
\end{array}\right) \int_{0}^{z}(z-t)^{K-1}(1+t)^{-(c+K)} d t .
$$

Majorizing the $R_{c, K}$ error terms by the respective integral over the absolute value of the integrand reduces to majorizing the error terms $\rho_{K}$ at $z=-\exp (-2 y)$, which is in $(-1,0)$ for any $y$ in $(A, B)$. From the analysis of the binomial series in this situation, change variables $x=-t$ in $\rho_{K}(z)$ and use the fact that $(|z|-x) /(1-x)$ is decreasing for $x$ in $[0,|z|]$ to obtain:

$$
\left|\rho_{K+1}\left(-e^{-2 y}\right)\right| \leq\left|1-\frac{1}{\left(1-e^{-2 A}\right)^{c}}\right|\left|\left(\begin{array}{c}
-(c+1) \\
K
\end{array}\right)\right| e^{-2 y K} .
$$

The estimate of the proposition follows on integration. Using Riemann's criterion, absolute convergence of the series obtained is implied by the right-hand side of the latter estimate going to 0 as $K$ tends to $\infty$. Indeed, using Stirling's formula, we have asymptotically

$$
\left|\left(\begin{array}{c}
-(c+1) \\
K
\end{array}\right)\right| J_{c, K}(g) \sim \frac{1}{\Gamma(|1+c|)} K^{\beta+c} \frac{J_{c, K}(g)}{K^{\beta}},
$$

as $K$ tends to $\infty$. From the $K$-asymptotics of $J_{c, K}(g)$, the error term $R_{c, K}$ thus goes to 0 as $K$ tends to $\infty$, and the proof of Proposition 6.13 is complete.

6.7.2. Proof of Theorem 6.4. This section shows how the asymptotic expansion for the imaginary parts function $I_{\kappa}(B, \infty)$ of Theorem 6.4 follows from the results of Section 6.7.1. In fact, checking with the Section 2.2 definitions we have identically:

$$
I_{\kappa}(B, \infty)=\operatorname{Im}\left(J_{B, \infty}\left(f_{\alpha, \gamma_{c},-a}\right)\right),
$$

on recalling that $\gamma_{c}=c /(2 \alpha)$. It is thus sufficient to construct asymptotic expansions for the $J$-integral. Applying Proposition 6.13 reduces this to the integrals $A_{\gamma, 2 n+\mu}(B)$, with $\gamma=\gamma_{c}+i(\pi / 2) \alpha$, defined by:

$$
e^{i a \frac{\pi}{2}} A_{\gamma, 2 n+\mu}(B)=J_{B, \infty, 2 n+\mu}\left(f_{\alpha, \gamma_{c},-a}\right) .
$$

A formal application of Proposition 6.13 then gives the desired series in the Section 6.1.2 functions $\operatorname{IEc}_{(\alpha, \delta, B)}(z)=\int_{[B, \infty]} \exp (-z y) \operatorname{Erfc}(\alpha y+\delta) d y$ using the identity:

$$
J_{B, \infty, 2 n+\mu, k}\left(f_{\alpha, \gamma_{c},-a}\right)=e^{i a \frac{\pi}{2}} \operatorname{IEc}_{(\alpha, \gamma, B)}((\mu+2 n)-a+2 k) .
$$

To verify the growth condition of Proposition 6.13 notice the majorization: $\left|\operatorname{IEc}_{\omega}(\eta)\right| \leq$ $\exp \left(\operatorname{Im}^{2}(\gamma)\right) \operatorname{IEc}_{\omega_{0}}(\operatorname{Re}(\eta))$, where we set $\omega=(\alpha, \gamma, B)$ and $\omega_{0}=\left(\alpha, \gamma_{c}, B\right)$. Referring to Remark 6.14, it is thus sufficient to establish exponential order decay to 0 of IEc $_{\omega_{0}}$ at $z_{m}=\operatorname{Re}(\mu-a)+2 m$ for $m$ tending to $\infty$. Taking $m$ so large that $z_{m}$ is positive,

$$
z_{m} \operatorname{IEc}_{\omega_{0}}\left(z_{m}\right)=e^{-z_{m} B} \operatorname{Erfc}\left(\alpha B+\gamma_{c}\right)-e^{-(\alpha B)^{2}-2 \alpha B\left(\gamma_{c}+\frac{z_{m}}{2 \alpha}\right)} \operatorname{WErfc}\left(\alpha B+\gamma_{c}+\frac{z_{m}}{2 \alpha}\right) .
$$

Here WErfc $(\eta)=\exp \left(\eta^{2}\right) \operatorname{Erfc}(\eta)$ is bounded on the reals; see [14, 1972, Section 2.3]. The asymptotic behaviour with $m$ tending to $\infty$ of the second summand above is hence dominated by that of its exponential factor too. Thus both summands exponentially decay to 0 with $m$ tending to $\infty$, and the proof of Theorem 6.4 is complete. 
6.8. Proof of the total real parts results. As a final step in establishing the Section 6 results this section develops representations and expansions for the Section 6.2 integrals over the vertical part of the Section 6.2 contour $L_{\pi / 2,0}$. This will establish the results for the total real parts contributions of Section 6.3. Conceptually, they give expression to the rôle of the hypergeometric function for this part of the formula of Theorem 4.1. Technically they depend on methods and results of Sections 6.7 and 6.8.

Recall $\bar{R}_{\kappa_{0}}=\sum_{\kappa \in\{\kappa( \pm a, \pm c)\}} R_{\kappa}(0)$ for $\kappa_{0}=(\mu, \beta, \alpha, a, c)$ with $\mu$ and $a$ real from Section 6.3, and, from Section 6.2, for any $\kappa=\left(\mu, \beta, \alpha, \kappa_{4}, \kappa_{5}\right)$ the defining integral:

$$
R_{\kappa}(0)=\int_{0}^{\pi / 2} \operatorname{Re}\left(H_{\mu}(-\beta \cosh (i \phi)) \exp \left(i \kappa_{4} \phi\right) \operatorname{Erfc}\left(\alpha i \phi+\kappa_{5} /(2 \alpha)\right)\right) d \phi .
$$

6.8.1. Integral representations. At the heart of the results of Section 6.3 is an integral representation as follows.

Proposition 6.15. In the above setting, $\bar{R}_{\kappa_{0}}=4 \int_{0}^{\pi / 2} H_{-\mu}(-\beta \cos \phi) \cos (a \phi) d \phi$.

REMARK 6.16. When $a=c$, as when computing Asian option values in Section 4.1,

$$
\left(R_{\kappa_{0}^{+}}+R_{\kappa_{0}^{-}}\right)(0)=(1 / 2) \bar{R}_{\kappa_{0}}=2 \int_{0}^{\pi / 2} H_{-\mu}(-\beta \cos \phi) \cos (a \phi) d \phi,
$$

where $\kappa_{0}^{ \pm}=\left(\mu, \beta, \kappa^{*}\right)$ with $\kappa^{*}=(\alpha, \pm(a, a))$.

The proposition gives expression to the behaviour of Erfc on the imaginary axis. Indeed, in the above integral for $R_{\kappa}(0)$ notice that $H_{-\mu}(-\beta \cosh (i \phi))=H_{-\mu}(-\beta \cos \phi)$, which is real. Then, for any complex variable $z=z_{0}+i z_{1}$, we have, on changing the contours of integration,

$$
\operatorname{Erfc}\left(z_{0}+i z_{1}\right)=\operatorname{Erfc}\left(z_{0}\right)-(2 / \sqrt{\pi}) \int_{\left[z_{0}, z_{0}+i z_{1}\right]} \exp \left(-w^{2}\right) d w .
$$

Significant simplifications, however, are thus obtained only by averaging over the $R_{\kappa}(0)$. Since $\operatorname{Erfc}(z)+\operatorname{Erfc}(-z)=2$, we in fact find after an explicit computation:

$$
\bar{R}_{\kappa_{0}}=4 \int_{0}^{\pi / 2} H_{-\mu}(-\beta \cos \phi) \cos (a \phi) d \phi-\frac{\exp \left(-(c /(2 \alpha))^{2}\right)}{\sqrt{\pi} / 2} \int_{0}^{\pi / 2} H_{-\mu}(\beta \cos \phi) \Sigma(\phi) d \phi,
$$

where

$$
\Sigma(\phi)=\int_{0}^{\phi} e^{+(\alpha x)^{2}} C(\phi, x) d x
$$

with

$$
C(\phi, x)=\sum_{\varepsilon_{1}, \varepsilon_{2} \in\{ \pm 1\}} \cos \left(\frac{\pi}{2}+\left(\varepsilon_{1} a \phi+\varepsilon_{2} \alpha x\right)\right) .
$$

The point is that $C(\phi, x)=0$ for all $\phi$ and $x$, which is immediate using $\cos (\pi / 2 \pm w)=$ $\mp \sin (w)$ for any real $w$. The proof of Proposition 6.15 is complete.

6.8.2. Proof of Theorem 6.1. This section establishes the integral representation for $\bar{R}_{\kappa_{0}}$ of Theorem 6.1 which is in terms of Kummer's function $\Phi$ and which asserts that:

$$
\bar{R}_{\kappa_{0}}=2 \pi \beta^{a} \frac{\Gamma((a+\mu) / 2)}{\Gamma(\mu) \Gamma(a+1)} \Phi\left(\frac{1}{2}(a+\mu), a+1 ; \beta^{2}\right)-4 K_{\lambda\left(\kappa_{0}\right)},
$$

where $K_{\lambda\left(\kappa_{0}\right)}=\int_{0}^{\infty} \operatorname{Im}\left(H_{-\mu}(-i \beta \sinh (y)) e^{-a\left(y+i \frac{\pi}{2}\right)}\right) d y$. 
The starting point is the representation $\bar{R}_{\kappa_{0}}=\int_{0}^{\pi / 2} H_{-\mu}(-\beta \cos \phi) \cos (a \phi) d \phi$ of Proposition 6.15 , which we consider as the limit with $\theta$ tending in $(\pi / 2, \pi]$ to $\pi / 2$ of the integrals:

$$
C_{\theta}=4 \int_{0}^{\theta} H_{-\mu}(-\beta \cos \phi) \cos (a \phi) d \phi .
$$

To compute any such $C_{\theta}$, express the Hermite function of its integrand in terms of the Section 2.1 integral. With $\cos \phi>0$, the integrand of the resulting double integral is integrable. Thus apply Fubini's theorem, interchange the order of integration, and get:

$$
C_{\theta}=(4 / \Gamma(\mu)) \int_{0}^{\infty} e^{-u^{2}} u^{\mu-1} \int_{0}^{\theta} \exp (2 u \beta \cos \phi) \cos (a \phi) d \phi d u .
$$

Interpret the inner integral here using the following form of the Schläfli representation of $I$-Bessel functions:

$$
\pi I_{\rho}(x)=\int_{0}^{\theta} e^{x \cos \phi} \cos (\rho \phi) d \phi+\int_{0}^{\infty} \operatorname{Im}(\exp (-\rho(i \theta+y)+x \cosh (i \theta+y))) d y,
$$

for any real $x>0$. This representation generalizes [30, 6 -22] and yields:

$$
C_{\theta}=(4 \pi / \Gamma(\mu)) A-4 B_{\theta},
$$

where

$$
\begin{gathered}
A=\int_{0}^{\infty} e^{-u^{2}} u^{\mu-1} I_{a}(2 \beta u) d u \\
B_{\theta}=\operatorname{Im}\left((1 / \Gamma(\mu)) \int_{0}^{\infty} e^{-u^{2}} u^{\mu-1} \int_{0}^{\infty} \exp (2 \beta u \cosh (i \theta+y)-a(i \theta+y)) d y d u\right) .
\end{gathered}
$$

These integrals are finite since $a+\mu>0$. The integral $A$ generalizes the classical Weber integral, and, proceeding as for [30, 13·3, p. 394] by expanding the Bessel function factor of $A$ 's integrand into its series and integrating term-by-term, we get:

$$
A=\frac{\pi}{2} \beta^{a} \frac{\Gamma(C)}{\Gamma(D)} \Phi\left(C, D ; \beta^{2}\right), \quad \text { where } \quad \Phi\left(C, D ; \beta^{2}\right)=\sum_{\ell=0}^{\infty}\left((C)_{\ell} /(D)_{\ell}\right) \frac{\beta^{2 \ell}}{\ell !},
$$

with $C=(a+\mu) / 2$ and $D=a+1$, as required.

With the respective integrands of $A$ and $B_{\theta}$ bounded as functions of $\theta$ in $[\pi / 2, \pi]$ and $y$ in $[0, \infty)$, taking the limit of $B_{\theta}$ for $\theta$ tending to $\pi / 2$ is effected by taking it under the integral sign. That $B_{\pi / 2}$ equals $K_{\lambda}$ follows on reversing the above interchange of integration. The proof of Theorem 6.1 is complete.

6.8.3. Error term expansions. Working in the setting of Section 6.3, this section provides the more general results mentioned there. For this, we study the role of the hypergeometric function ${ }_{2} F_{1}$ for the partial error terms $K_{\lambda}(B)$, given by:

$$
K_{\lambda}(B)=\int_{B}^{\infty} \operatorname{Im}\left(H_{-\mu}(-i \beta \sinh (y)) \exp \left(-a\left(y+i \frac{\pi}{2}\right)\right)\right) d y,
$$

for any real $B \geq 0$. Recall that in $\lambda=(\mu, \beta,(\alpha, a))$ we take $\mu>0$ and $a$ real and let $a+\mu>0$, so that $K_{\lambda}(B)$ is finite. Our main finding is a two-step asymptotic expansion for these terms. Its first step effects a reduction to the integrals $D_{b, d}(B)$ given by

$$
D_{b, d}(B)=\int_{B}^{\infty} \frac{\exp (-b y)}{(2 \sinh (y))^{d}} d y
$$

for any complex numbers $b, d$ such that $\operatorname{Re}(b+d)>0$. The precise result is as follows. 
ThEOREm 6.17. In the above setting, where $\mu$ and $a+\mu$ are positive, we have for any integer $n_{0} \geq 0$ the expansion

$$
K_{\lambda}(B)=\sum_{n=0}^{n_{0}-1} \frac{(-1)^{n} b_{n}}{\beta^{2 n+\mu}} \operatorname{Im}\left(e^{i \frac{\pi}{2}(\mu-a)} D_{a, 2 n+\mu}(B)\right)+\rho_{n_{0}}(B),
$$

where $b_{n}=b_{n}(\mu)$ are as in Section 6.1.3, and where the error terms $\rho_{n_{0}}(B)$ satisfy

$$
\left|\rho_{n_{0}}(B)\right| \leq\left|b_{n_{0}}\right|(\sqrt{2} / \beta)^{2 n_{0}+\mu} D_{a, 2 n_{0}+\mu}(B) .
$$

Postponing the proof briefly, this result leaves the task of computing the $D$-integrals. Here ${ }_{2} F_{1}$ enters, and as a second step, we have the following result.

Theorem 6.18. If $\operatorname{Re}(b+d)>0$, we have for $B$ any positive real:

$$
D_{b, d}(B)=\frac{\exp (-(b+d) B)}{(b+d)(1-\exp (-2 B))^{d-1}}{ }_{2} F_{1}(-M, 1 ; P ; \exp (-2 B)),
$$

where $M=(d-b) / 2-1$ and $P=1+(b+d) / 2$. If moreover $d-b \geq 2$ is an even integer,

$$
D_{b, d}(B)=\frac{1}{(b+d)(1-\exp (-2 B))^{d-1}} \sum_{m=0}^{M} \frac{(-M)_{m}}{(P)_{m}} \exp (-(2 m+b+d) B) .
$$

Proof of Theorem 6.17. We proceed by reduction to Proposition 6.13. In fact, we have $K_{\lambda}(B)=\operatorname{Im}\left(J_{B, \infty}\left(f_{a}\right)\right)$ for the function $f_{a}(w)=\exp (-a w)$, so that:

$$
J_{B, \infty}\left(f_{a}\right)=\sum_{n=0}^{n_{0}-1} \frac{(-1)^{n} b_{n}}{\beta^{2 n+\mu}} e^{i \frac{\pi}{2} \mu} J_{B, \infty, 2 n+\mu}\left(f_{a}\right)+\rho_{n_{0}}\left(f_{a}\right) .
$$

Here the error terms $\rho_{n_{0}}\left(f_{a}\right)$ satisfy

$$
\left|\rho_{n_{0}}\left(f_{a}\right)\right| \leq\left|b_{n_{0}}\right|(\sqrt{2} / \beta)^{2 n_{0}+\mu} J_{B, \infty, 2 n_{0}+\mu}\left(\left|f_{a}\right|\right) .
$$

Since we have $J_{B, \infty, 2 n+\mu}\left(f_{a}\right)=\exp (-i(\pi / 2) a) D_{a, d}(B)$ by construction, the proof of Theorem 6.17 is complete.

The proof of the second result needs three facts about ${ }_{2} F_{1}$ to be recalled first, referring to [14, Chapter 9] as well as [11, Chapter II] and [1, Chapter 15]. For any complex numbers $a, b, c$ with $c$ not in $\mathbf{Z}_{\leq 0}$ the function is defined by the series:

$$
{ }_{2} F_{1}(a, b ; c ; z)=\sum_{n=0}^{\infty}\left((a)_{n}(b)_{n}\right) /\left((c)_{n} n !\right) z^{n} \quad \text { for }|z|<1 .
$$

If $0<\operatorname{Re} b<\operatorname{Re} c$, we have, for any $z$ in $\mathbf{C} \backslash[1, \infty)$, Euler's integral representation:

$$
{ }_{2} F_{1}(a, b ; c ; z)=(\Gamma(c) /(\Gamma(b) \Gamma(c-b))) \int_{0}^{1} w^{b-1}(1-w)^{c-b-1}(1-z w)^{-a} d w .
$$

For such a $z$, also recall the linear relation:

$$
{ }_{2} F_{1}(a, b ; c ; z)=(1-z){ }_{2}^{c-a-b} F_{1}(c-a, c-b ; c ; z) ;
$$

see [14, Equation (9.5.3)].

Proof of Theorem 6.18. Simplifying notation by putting $E=(b+d) / 2$, so that we have $P=E+1$ in the integral for $D_{b, d}(B)$, successively change variables $x=\exp (-2 y)$ and $w=x \exp (2 B)$ to get as a first step:

$$
D_{b, d}(B)=\frac{\exp (-(b+d) B)}{2} \int_{1}^{0} \frac{w^{E-1}}{\left(1-e^{-2 B} w\right)^{d}} d w .
$$


As a second step, we verify for any $z$ not in $[1, \infty)$ the identity:

$$
\frac{1}{2} \int_{1}^{0} \frac{w^{E-1}}{(1-z w)^{d}} d w=\frac{1}{b+d} \frac{1}{(1-z)^{d-1}}{ }_{2} F_{1}(-M, 1 ; P ; z) .
$$

Indeed, ${ }_{2} F_{1}(\alpha, \beta ; \gamma ; z)$ has, for the parameters $\alpha=d, \beta=E, \gamma=\beta+1=P$, from the above the integral representation: ${ }_{2} F_{1}(d, E ; P ; z)=E \int_{0}^{1} w^{E-1} /(1-z w)^{d} d w$. Apply the above linear relation to complete the proof of the second step.

At this point, moreover assume: $d-b \geq 2$ is an even integer. Then any Pochhammer symbol $(-M)_{n}$ is 0 for $n \geq 1+M$. Thus the series in step two for the hypergeometric function is a polynomial: ${ }_{2} F_{1}(-M, 1 ; P ; z)=\sum_{n=0}^{M}\left((-M)_{n} /(P)_{n}\right) z^{n}$ for any $z$ not in $[1, \infty)$. Using the identity of the second step, with $z$ equal to $\exp (-2 B)$ in the identity of the first step, then completes the proof of Theorem 6.18.

6.8.4. Proof of Theorem 6.2. The proof of Theorem 6.2 is effected by combining Theorem 6.11 and Theorem 6.17. For this decompose the defining integral of $K_{\lambda}$ at any real $B>0$ to obtain with the concepts of Sections 6.6.2 and 6.8:

$$
K_{\lambda}=\operatorname{Im}\left(e^{-i \frac{\pi}{2} a} I_{0, B}\left(f_{a}\right)\right)+K_{\lambda}(B), \quad \text { where } \quad f_{a}(y)=\exp (-a y) .
$$

The second summand then expands according to Theorem 6.17 . Taking $n_{0}=0$ there, we have $\left|K_{\lambda}(B)\right| \leq\left(2^{1 / 2} / \beta\right)^{\mu} D_{a_{0}, \mu}(B)$, with the $D$-factor computed by the second formula of Theorem 6.18 .

The first summand of the above sum, on the other hand, expands according to Theorem 6.11. Taking $n_{0}=0$ there, one finds $\left|\rho_{0}\left(f_{a}\right)\right| \leq\left|a_{0}(\mu)\right| \Delta_{0, B, 0}^{0}\left(\left|f_{a}\right|\right)$. Here, $2\left|a_{0}(\mu)\right|=\Gamma(\mu / 2) / \Gamma(\mu)$ from Section 6.1.3, and $\Delta_{0, B, 0}^{0}\left(\left|f_{a}\right|\right)=2(1-\exp (-a B)) / a$ by a direct computation. This completes the proof of Theorem 6.2, making clear the general expansion behind this result. The proofs of the Section 6 results thus are also complete.

7. Numerical Asian option valuation. Computing Asian option values using a form of the law as discussed in Remark 4.2 has consistently been hindered by the structure of these representations. A number of such severe problems now are detailed in [3]; see also [6, Section 5]. This section demonstrates how the Section 4 and 6 results now enable a benchmark valuation of Asian options in the Black-Scholes setting.

7.1. Principal remarks on normalized valuation. The point of working with normalized values $C^{(\nu)}$ is that computation of the time- $t$ values $C_{t}$ of any Asian option can be reduced to this single dimensionless function of three dimensionless variables. This is by way of the Section 3.2 factorization

$$
C_{t}=S_{t} \operatorname{norm}_{\sigma, t} C^{(\nu)}(h, q), \quad \text { where } \quad \operatorname{norm}_{\sigma, t}=\frac{\exp (-r(T-t))}{(\sigma / 2)^{2}\left(T-t_{0}\right)} .
$$

The cost of this simplification, however, is that normalized values have to be implemented at higher accuracies for this reduction to be useful; see [24, Section 7.2] for more detail. The results of our paper now enable the computation of normalized values to, in principle, any accuracy, and thus enable a benchmark valuation of Asian options in the mathematically strict sense. We demonstrate this point of view in Section 7.2. 


\begin{tabular}{|c|c|}
\hline$\sigma$ & $\gamma_{\nu, q} R$ \\
\hline $20 \%$ & 0.00046380930057892 \\
\hline $30 \%$ & 0.00104357092629580 \\
\hline $40 \%$ & 0.00185524580297281 \\
\hline $50 \%$ & 0.00290260933724007 \\
\hline
\end{tabular}

TABLE 7.1. Total real part contributions.

\begin{tabular}{|c|c|c|c|c|}
\hline & $T=1$ year & $T=9$ months & $T=6$ months & $T=3$ months \\
\hline$\sigma=20 \%$ & $27 \mathrm{D}$ & $34 \mathrm{D}$ & $50 \mathrm{D}$ & $94 \mathrm{D}$ \\
\hline$\sigma=30 \%$ & $12 \mathrm{D}$ & $16 \mathrm{D}$ & $22 \mathrm{D}$ & $42 \mathrm{D}$ \\
\hline$\sigma=40 \%$ & $7 \mathrm{D}$ & $9 \mathrm{D}$ & $13 \mathrm{D}$ & $24 \mathrm{D}$ \\
\hline$\sigma=50 \%$ & $4 \mathrm{D}$ & $6 \mathrm{D}$ & $8 \mathrm{D}$ & $15 \mathrm{D}$ \\
\hline
\end{tabular}

TABLE 7.2. Minimal number of correct decimal places of the Corollary 6.3 quasi-closed form $\gamma_{\nu, q} R_{\text {est }}$ as a function of $\sigma$ and $T$.

7.2. Numerical examples. This section numerically illustrates the results of Section 6. This is done by considering the Section 3.2 normalized valuation of an Asian option on a non-dividend-paying stock which is issued at time 0 at the money: $K=S_{0}$. Then we have $q=h=(\sigma / 2)^{2} T$. We let $T=1$ and assume an interest rate of $r=9 / 100$ in $\nu=2 r / \sigma^{2}-1$. The Section 3.2 normalized values we represent as follows:

$$
C^{(\nu)}(h, q)=\gamma_{\nu, q}(R+I)
$$

with

$$
2 R=\exp (2 h(\nu+1)) \bar{R}_{\kappa(h, \nu+2)}-\bar{R}_{\kappa(h, \nu)},
$$

the total real part contribution, and $I$ the total imaginary part contribution given by:

$$
I=\exp (2 h(\nu+1)) \sum_{\kappa \in K(\xi(h, \nu+2))} I_{\kappa}(0, \infty)-\sum_{\kappa \in K(\xi(h, \nu))} I_{\kappa}(0, \infty) .
$$

Seeking results of an accuracy of 15 places after the decimal point (15D), all computations have been done on an HP Visualize C3600 workstation using GP/PARI CALCULATOR Version 2.1.0 and Mathematica 4.1.

With the values of $R$ recorded in Table 7.1 against $\sigma$ varying from $20 \%$ p.a. to $50 \%$ p.a., we concentrate in the present paper on the approximation qualities of the quasiclosed form $R_{\text {est }}$ of Corollary 6.3. An estimate for the error term $\Delta$ there is afforded by the majorizations of Theorem 6.2 for its single summands. We thus obtain the minimal accuracies with which $\gamma_{\nu, q} R_{\text {est }}$ approximates $\gamma_{\nu, q} R$ as recorded in Table 7.2 in dependency on time to maturity $T$ and $\sigma$. The values of $R$ themselves have been computed using a series we have derived in [22], which is omitted from the present paper for reasons of space. Using this series, and using the Corollary 6.3 quasi-closed form $R_{\text {est }}$, actually appears to furnish complementary concepts for computing $R$.

We obtain $I$ by computing separately the respective four single summands corresponding to $c$ in $\{ \pm \nu\}$ and $\{ \pm(\nu+2)\}$. Our values for it are summarized in the last column 


\begin{tabular}{|c|c|c|c|c|c|}
\hline$\sigma$ & $B$ & $N_{a e}$ & $N_{s}$ & maximal error & $\gamma_{\nu, q} I$ \\
\hline $20 \%$ & 1.066 & 34 & 532 & $4.782 \times 10^{-16}$ & 0.000277750687304 \\
\hline $30 \%$ & 1.22 & $(19,22)$ & 345 & $4.877 \times 10^{-16}$ & 0.001129974119954 \\
\hline $40 \%$ & 1.395 & 19 & 289 & $4.787 \times 10^{-16}$ & 0.002925757480443 \\
\hline $50 \%$ & 1.572 & 18 & 275 & $4.827 \times 10^{-16}$ & 0.006006811114892 \\
\hline
\end{tabular}

TABLE 7.3. Total imaginary part contributions.

\begin{tabular}{|c|c|c|}
\hline$\sigma$ & maximal error & $C^{(\nu)}(h, q)$ \\
\hline $20 \%$ & $4.9727 \times 10^{-16}$ & 0.00074155998788343 \\
\hline $30 \%$ & $4.9687 \times 10^{-16}$ & 0.00217354504625037 \\
\hline $40 \%$ & $4.9157 \times 10^{-16}$ & 0.00478100328341654 \\
\hline $50 \%$ & $4.9461 \times 10^{-16}$ & 0.00890942045213227 \\
\hline
\end{tabular}

TABLE 7.4. Normalized values $C^{(\nu)}(h, q)$ of the Asian option for $T=1$.

of Table 7.3 against the volatility $\sigma$. Proceeding along the lines described in Section 6.4, start with the asymptotic expansions of Theorem 6.4. First use the error estimates there to determine for each single of its four terms break points $B$ as small as possible such that the sum of the four weighted error terms thus obtained is below the desired accuracy too. Identical break points $B$ were thus arrived at, which are recorded in the second column of Table 7.3. The third and the fourth columns of this table then give the number of terms $N_{a e}$ of the Theorem 6.4 asymptotic expansion and the number of terms $N_{s}$ of the Theorem 6.6 series we have used in our computations, with the latter terms determined using the error estimates of Theorem 6.6. The minimal accuracies for the values of $I$ thus achieved are recorded in the next to last column. Due to the vintage of our machine and the high accuracies with which we computed the error function values needed using Mathematica, computation times for this table were in the minutes range.

Combining these results, we thus obtain the values of $C^{(\nu)}(h, q)$ against $\sigma$ recorded in the last column of Table 7.4 to the minimal accuracies given in its second column. We have confirmed these results in [24, Section 9] and [26, Section 8] using alternative methods. Referring to Section 7.1, implementing the function $C^{(\nu)}$ along the above lines will hence allow making efficient use of the Section 6 results.

7.3. Alternative approaches. To compare with the more recent literature, the results of this paper's approach steer a middle course between those of [8] and [24], on the one hand, and [15], on the other hand. The latter paper seeks to develop a two-step approximation approach to Asian option values based on a spectral analysis of the infinitesimal generator of $A^{(\nu)}$ along the lines of [17]; its results seem to be obtained as excruciatingly hard as they are experimental.

Referring to [26, Section 8] for a comparison of the two Laguerre reduction series approaches of [24, 26], and 8, a comparison with the present paper's approach has to view two sides. On the one hand, the convergence of our paper's expansions in error functions is not as fast as that of the series of [24], which are in terms of error 
functions as well. On the other hand, the error estimates of the latter paper are not as explicit and sharp as those of the present one. With our two papers' computations based on their respective error estimates, however, our results should be capable of confirmation by independent calculation, and they moreover provide benchmarks in the strict mathematical sense.

8. Epilogue. The paper has concentrated on the rôle of complex analytic methods in valuing contingent claims which we have demonstrated in the case of Asian options. As a first step, the Laplace transform was used to get an integral representation of their values. A dimension reduction of the problem has thus been achieved in particular, a fact that holds on a principal level for values of Asian options in general Lévy process models; see [25]. This has then enabled us to derive implementable explicit formulas for computation as a second step, combining series with asymptotic expansions. It seems noteworthy to what extent these questions are connected with hypergeometric and confluent hypergeometric functions. Indicative of this are in particular the results proved in Section 6.8 which furnish quasi-closed forms for the real parts of integrals for Asian option values. Further work in connecting finance and constructive complex analysis may thus be worthwhile.

\section{REFERENCES}

[1] M. Abramowitz and I.A. Stegun, Handbook of mathematical functions with formulas, graphs, and mathematical tables, Dover Publications, New York, 1964. MR0167642 (29:4914)

[2] R. Beals, Advanced mathematical analysis, Springer, New York, 1973. MR0530403 (58:26624)

[3] P. Barrieu, A. Rouault, and M. Yor, A study of the Hartman-Watson distribution motivated by numerical problems related to the pricing of Asian options, J. Appl. Prob. 41 (2003), 1049-1058. MR 2122799 (2006a:60032)

[4] P. Carr, Personal communication, November 1999.

[5] P. Carr and M. Schröder, On the valuation of arithmetic-average Asian options: The Geman-Yor Laplace transform revisited, manuscript 24pp., 2000.

[6] P. Carr and M. Schröder, Bessel processes, the integral of geometric Brownian motion, and Asian options, Theorija verojatnostej i ee primenenija 48, 503-533 (2003); English version in, Theory of probability and its applications 48, 400-425 (2004). MR2141348(2006e:60119)

[7] G. Doetsch, Handbuch der Laplace-Transformation. Band I, Birkhäuser Verlag, Basel, 1971. MR 0344807 (49:9546)

[8] D. Dufresne, Laguerre series for Asian and other options, Math. Finance 10 (2000), 407-428. MR.1785163(2001i:91058)

[9] D. Dufresne, The integral of geometric Brownian motion, Adv. Appl. Prob. 33 (2001), 223-241. MR:1825324 (2002c:60132)

[10] D. Dufresne, Bessel processes and Asian options, Numerical Methods in Finance (M. Breton and H. Ben-Ameur, eds.), Springer, New York, 2005, pp. 35-57. MR2180792 (2006e:91005)

[11] A. Erdélyi, W. Magnus, F. Oberhettinger, and F. Tricomi, Higher transcendental functions, R.E. Krieger Publ., Malabar, 1953, reprinted 1981. MR0058756 (15:419i)

[12] H. Geman and M. Yor, Bessel processes, Asian options, and perpetuities, Math. Finance 3 (1993), 349-375.

[13] D. Lamberton and B. Lapeyre, Introduction to stochastic calculus applied to finance, Chapman and Hall, London, 1996. MR:1422250 (98b:90018)

[14] N.N. Lebedev, Special functions and their applications, Dover Publications, New York, 1971. MR0350075 (50:2568)

[15] V. Linetsky, Spectral expansions for Asian options, Oper. Res. 52 (2004), 856-867. MR.2104142 (2005i:60156)

[16] A. Lipton-Lifschitz, Similarities via self-similarities, RISK (September 1999), 101-105. 
[17] H.P. McKean, Elementary solutions of certain parabolic differential equations, Transactions AMS 82 (1956), 519-548. MR0087012 (19:285b)

[18] F.W.J. Olver, Uniform asymptotic expansions for Weber parabolic cylinder functions of large orders, J. Res. Nat. Bur. Standards 63B (1959), 131-169. MR0109898 (22:781)

[19] F.W.J. Olver, Uniform, exponentially improved, asymptotic expansions for the confluent hypergeometric function and other integral transforms, SIAM J. Math. Anal. 22 (1991), 1475-1489. MR 1112521 (92g:41035)

[20] F.W.J. Olver, Asymptotics and special functions, Reprint of the 1974 original, A.K. Peters, Wellesley, MA, 1997. MR.1429619 (97i:41001)

[21] L.C.G. Rogers and Z. Shi, The value of an Asian option, J. Appl. Prob. 32 (1995), 1077-1088. MR:1363350 (96j:90017)

[22] M. Schröder, Mathematical ramifications of option valuation: The case of the Asian option, Habilitationsschrift, Universität Mannheim, April 2002.

[23] M. Schröder, On the integral of geometric Brownian motion, Adv. Appl. Prob. 35 (2003), 159-183. MR:1975509 (2004b:60202)

[24] M. Schröder, Laguerre series in contingent claim valuation, with applications to Asian options, Mathematical Finance 15 (2005), 491-531. MR2147159 (2006h:91084)

[25] M. Schröder, A constructive Hartman-Watson approach to stochastic functionals, with applications to finance, 2005.

[26] M. Schröder, On ladder height densities and Laguerre series in the study of stochastic functionals. II: Exponential functionals of Brownian motion and Asian option values, Adv. Appl. Prob. 38 (2006), 995-1027. MR2285691

[27] J.M. Steele, Stochastic calculus and financial applications, Springer, New York, 2000. MR1783083 (2001i:60080)

[28] N. Temme, Numerical and asymptotic aspects of parabolic cylinder functions, J. Comput. Appl. Math. 121 (2000), 221-246. MR1780050 (2002a:33007)

[29] R. Vidunas and N. Temme, Parabolic cylinder functions: Examples of error bounds for asymptotic expansions, CWI Report MAS-R0225, 2002. MR1993339 (2004e:41040)

[30] G.N. Watson, A treatise on the theory of Bessel functions, Cambridge University Press, Cambridge, 2nd rev ed. 1944, reprinted 1995. MR0010746 (6:64a)

[31] E.T. Whittaker and G.N. Watson, A course of modern analysis, Cambridge University Press, Cambridge, 1927; 4th ed. 1962. MR0178117 (31:2375)

[32] M. Yor, Loi de l'indice du lacet Brownien, et distribution de Hartman-Watson, Z. Wahrscheinlichkeitstheorie 53 (1980), 71-95. MR576898 (82a:60120)

[33] M. Yor, On some exponential functionals of Brownian motion, Adv. Appl. Prob. 24 (1992), 509531. MR.1174378 (94b:60095)

[34] M. Yor, Exponential functionals and principal values related to Brownian motion, Biblioteca de la revista mathemática iberoamericana., Madrid, 1997. MR1648653 (99d:60003)

[35] M. Yor, Exponential functionals of Brownian motion and related processes, Springer, Heidelberg, 2001. MR 1854494 (2003f:60147) 